\title{
CLASSICAL AND ELLIPTIC POLYLOGARITHMS AND SPECIAL VALUES OF L-SERIES
}

\author{
Don Zagier AND HeRBert GANGL ${ }^{1}$
}

The Dirichlet class number formula expresses the residue at $s=1$ of the Dedekind zeta function $\zeta_{F}(s)$ of an arbitrary algebraic number field $F$ as the product of a simple factor (involving the class number of the field) with the determinant of a matrix whose entries are logarithms of units in the field. On the other hand, if $F$ is a totally real number field of degree $n$, then a famous theorem by Klingen and Siegel says that the value $\zeta_{F}(m)$ for every positive even integer $m$ is a rational multiple of $\pi^{m n}$. In [52] and [53], a conjectural generalization of these two results was formulated according to which the special value $\zeta_{F}(m)$ for arbitrary number fields $F$ and positive integers $m$ can be expressed in terms of special values of a transcendental function depending only on $m$, namely the $m^{\text {th }}$ classical polylogarithm function. These instances are expected to form part of a much more general picture in which a special value of an $L$-series of "motivic origin" is expressed in terms of some transcendental function. In this survey we collect some pieces fitting into and illustrating this picture.

The paper is organized as follows.

In Part I we review the polylogarithm conjecture and survey some of the known results. The case $m=2$, which is related to volumes of hyperbolic manifolds and has a more geometric flavor, is discussed in detail in $\S 1$. In the next two sections we formulate the conjecture for general $m$ and describe some of the numerical and theoretical evidence which supports it, while in $\S 4$ we discuss a refinement involving a "lifting" from $\mathbb{R}$ to $\mathbb{C} /(2 \pi i)^{m} \mathbb{Q}$ of the $m^{\text {th }}$ polylogarithm function. The natural setting for all of this is algebraic $K$-theory and the conjectures about polylogarithms lead to a purely algebraic (conjectural) description of the higher $K$-groups of fields.

In Part II we simultaneously generalize and specialize by considering partial zeta functions $\zeta_{F, \mathcal{A}}(s)$ (i.e. the analogue for $m \geq 1$ of the famous Stark conjectures about values of $L$-series at $s=1$ ) but restricting our attention to the case where $F$ is an imaginary quadratic field. This case, which for $m=1$ is the Kronecker limit formula, can be treated much more concretely than the general case, both experimentally and theoretically. We describe experimental computations for small $m$ and results of Deninger and Levin for $m=2$. An interesting aspect here is that the existence of the above-mentioned "lifting" of the polylogarithms suggests that the partial zeta-values $D_{F}^{m-1 / 2} \zeta_{F, \mathcal{A}}(m)$ should also have a natural lifting to $\mathbb{C} / \pi^{2 m} \mathbb{Q}$. It turns out that this "lifted" partial zeta-value really can be defined in a natural way (using Eisenstein series) and that the natural conjecture "lifted zeta-value = lifted polylog value" is supported by the numerical data (cf. $\S 6$ ). In this way the unproved polylogarithm conjecture leads to a definition of a new invariant for ideal classes in imaginary quadratic number fields.

Finally, in Part III we describe the numerical and theoretical evidence for conjectures expressing special values of $L$-series associated to elliptic curves in terms of "elliptic polylogarithm functions" which are obtained by an averaging process from the classical polylogarithms. Again the natural way to understand the results is in terms of algebraic $K$-theory, this time of the elliptic curve. We also describe particularly interesting examples of the conjectural picture coming from the recent work of Deninger, Boyd and Villegas on Mahler measures.

\footnotetext{
${ }^{1}$ Text based on the lectures given by D. Zagier at Banff
} 


\section{Part I. Polylogarithms, zeta-Values, and algebraic $K$-theory}

\section{Dilogarithms, hyperbolic manifolds, $\zeta_{F}(2)$ and $K_{3}(F)$}

Imaginary quadratic fields and Humbert's formula. We first consider the case of an imaginary quadratic field $F=\mathbb{Q}(\sqrt{-d})$ of discriminant $-d$. The group $S L_{2}\left(\mathcal{O}_{F}\right)$ acts as a discrete group of isometries on the hyperbolic 3 -space $\mathbb{H}^{3}$, and it is well-known that

$$
\zeta_{F}(2)=\frac{4 \pi^{2}}{d \sqrt{d}} \operatorname{Vol}\left(\mathbb{H}^{3} / S L_{2}\left(\mathcal{O}_{F}\right)\right)
$$

(originally found by Humbert - modulo a gap which can be corrected [28] —in 1919, and nowadays proved with the help of Tamagawa numbers or residues of Eisenstein series).

On the other hand, any hyperbolic 3-dimensional manifold can be triangulated, and we can assume that the vertices of the tetrahedra are algebraic numbers. By a formula of Lobachevsky (as given in detail in Thurston/Milnor [46]), the volume of a general hyperbolic tetrahedron can be expressed as a combination of a fixed number (24, to be precise) of values of the Bloch-Wigner dilogarithm

$$
D(z)=\Im\left(L i_{2}(z)+\log |z| \log (1-z)\right),
$$

evaluated at arguments which are algebraic functions of the coordinates of the vertices; here $L i_{2}$ is the dilogarithm function, which is given in the unit disk by

$$
L i_{2}(z)=\sum_{n>0} \frac{z^{n}}{n^{2}}, \quad|z|<1,
$$

and can be extended analytically to a multivalued function on $\mathbb{C}-\{0,1\}$, while the modified function $D(z)$ turns out to be one-valued.

Combining these two facts, we can express $\zeta_{F}(2)$ in terms of $D(z)$ with algebraic arguments $z$. As an example, we give the case $F=\mathbb{Q}(\sqrt{-7})$. Here various descriptions of the manifold $\mathbb{H}^{3} / \Gamma$ for some torsionfree subgroup $\Gamma \subset S L_{2}\left(\mathcal{O}_{F}\right)$ (as a link complement, as a union of two hyperbolic prisms, etc.) are given in [46]. We can use them to compute the volume and then, combining with Humbert's formula as given above, obtain e.g.

$$
\zeta_{\mathbb{Q}(\sqrt{-7})}(2)=\frac{4 \pi^{2} / 3}{7^{3 / 2}} D\left(\xi_{7}\right), \quad \text { where } \quad \xi_{7}=2\left[\frac{1+\sqrt{-7}}{2}\right]+\left[\frac{-1+\sqrt{-7}}{4}\right] .
$$

(Here and in the following we use the evident linear extension of a function on a set $X$ to a function on $\mathbb{Z}[X]$.)

For other fields the formulas quickly become rather complicated, e.g.

$$
\zeta_{\mathbb{Q}(\sqrt{-23})}(2)=\frac{4 \pi^{2} / 3}{23^{3 / 2}} D\left(\xi_{23}\right),
$$

with

$$
\xi_{23}=21\left[\frac{1+\sqrt{-23}}{2}\right]+7[2+\sqrt{-23}]+\left[\frac{3+\sqrt{-23}}{2}\right]-3\left[\frac{5+\sqrt{-23}}{2}\right]+[3+\sqrt{-23}] .
$$


General number fields. Now let $F$ be a general number field of discriminant $D_{F}$ and degree $[F: \mathbb{Q}]=n=r_{1}+2 r_{2}$. If the number $r_{2}$ of pairs of complex embeddings of $F$ equals 1, then a similar argument applies: we can associate to $F$ a certain discrete subgroup $\Gamma \subset S L_{2}(\mathbb{C})$ and a manifold (now even compact) $M=\mathbb{H}^{3} / \Gamma$, with the property that $\operatorname{Vol}(M) \sim_{\mathbb{Q}^{x}}$ $\left|D_{F}\right|^{1 / 2} \pi^{-2(n-1)} \zeta_{F}(2)$, where $\sim_{\mathbb{Q}^{\times}}$means that the term on the right is a non-zero rational multiple of the one on the left. (Take $\Gamma$ to be the group of units of an order in a quaternion algebra over $F$ ramified at all real places.) We again deduce that $\left|D_{F}\right|^{1 / 2} \pi^{-2(n-1)} \zeta_{F}(2)$ is a $\mathbb{Q}$-linear combination of values of $D(x)$ with $x \in \overline{\mathbb{Q}}$.

For $r_{2}>1$, we can get in the same way a discrete subgroup $\Gamma$ of $S L_{2}(\mathbb{C})^{r_{2}}$ such that the quotient $M:=\left(\mathbb{H}^{3}\right)^{r_{2}} / \Gamma$ has volume a rational multiple of $\left|D_{F}\right|^{1 / 2} \pi^{-2\left(r_{1}+r_{2}\right)} \zeta_{F}(2)$. We then use a lemma which asserts that such a quotient can be triangulated into a finite union of products $\Delta_{1} \times \cdots \times \Delta_{r_{2}}$ where each $\Delta_{i}$ is a tetrahedron in $\mathbb{H}^{3}$; the volume is therefore expressible as a finite sum of $r_{2}$-fold products of $D$ 's with algebraic arguments. The details are given in [52].

The Bloch group. Any triangulation of a complete hyperbolic 3-manifold $M$ provides, together with the above-mentioned formula for the volume of a hyperbolic 3 -simplex, an expression for the volume of $M$ in terms of dilogarithms,

$$
\operatorname{Vol}(M)=\sum_{i=1}^{N} D\left(z_{i}\right)
$$

for some $z_{i} \in \mathbb{C}$ (we can assume the $z_{i}$ to actually lie in $\overline{\mathbb{Q}}$, considered as a subfield of $\mathbb{C}$ ).

An analysis of the triangulation yields that the $z_{i}$ occurring in the formula are always subject to the algebraic condition

$$
\sum_{i} z_{i} \wedge\left(1-z_{i}\right)=0 \in \bigwedge^{2} \mathbb{C}^{\times}
$$

This follows, for example, from the combinatorial description of triangulations in [37] or from the results of [18].

We reformulate this fact by stating that each pair (manifold, triangulation) produces an element $\xi \in \mathcal{A}(\overline{\mathbb{Q}})$ with $\operatorname{Vol}(M)=D(\xi)$, where we define for any field $F$

$$
\mathcal{A}(F)=\left\{\sum n_{i}\left[z_{i}\right] \in \mathbb{Z}[F] \mid \sum n_{i}\left(z_{i} \wedge\left(1-z_{i}\right)\right)=0\right\}
$$

(here [1] and [0] are to be interpreted as belonging to $\mathcal{A}(F)$ ) and the function $D: \mathbb{C} \rightarrow \mathbb{R}$ is extended to $\mathbb{Z}[\mathbb{C}]$ by linearity as above. What happens when we change the triangulation? We will get different $z_{i}$ 's and a different expression for the volume but the two expressions will be obtained from one another by a finite number of applications of the five term relation

$$
D(x)+D(y)+D\left(\frac{1-x}{1-x y}\right)+D(1-x y)+D\left(\frac{1-y}{1-x y}\right) .
$$

This relation, which was discovered many times in the $19^{\text {th }}$ century (in the more complicated version using $L i_{2}$ rather than $D$ ), can be verified easily by differentiating it (w.r.t. $x$, say), since the derivative of $D(z)$ is an elementary function, but there is a prettier geometric way. The value of a general hyperbolic tetrahedron, as already mentioned, is a sum of $24 D$-values, but the volume of an ideal tetrahedron (one with all its vertices in $\partial \overline{\mathbb{H}^{3}} \cong \mathbb{P}^{1}(\mathbb{C})$ ) is equal to a single value of $D$, namely $D(\mathrm{r}(a, b, c, d))$ where $a, b, c, d \in \mathbb{P}^{1}(\mathbb{C})$ are the vertices and $\mathrm{r}$ denotes the cross-ratio. Now if we take five points $a_{1}, \ldots, a_{5}$ in $\mathbb{P}^{1}(\mathbb{C})$, then the "signed volume"

$$
\sum_{i=1}^{5}(-1)^{i} \operatorname{Vol}\left(\Delta\left(a_{1}, \ldots, \widehat{a_{i}}, \ldots, a_{5}\right)\right)=\sum_{i=1}^{5}(-1)^{i} D\left(\mathrm{r}\left(a_{1}, \ldots, \widehat{a_{i}}, \ldots, a_{5}\right)\right)
$$


vanishes, and this is equivalent to the five term relation. This is a special configuration, but one can show that the effect on the volume formula of subdividing any hyperbolic simplex into smaller pieces is also a consequence of the five term relation.

These considerations motivate defining, for any field $F$, the Bloch group $\mathcal{B}(F)$ as the quotient

$$
\mathcal{B}(F)=\frac{\mathcal{A}(F)}{\mathcal{C}(F)}
$$

where $\mathcal{C}(F)$ is the group generated by the 5 -term relation, i.e.

$$
\mathcal{C}(F)=\left\{[x]+[y]+\left[\frac{1-x}{1-x y}\right]+[1-x y]+\left[\frac{1-y}{1-x y}\right] \mid x, y \in F, x y \neq 1\right\} .
$$

(The reader should check that $\mathcal{C}(F) \subset \mathcal{A}(F)$.) By (2), it is obvious that $D$ is a well-defined linear function from $\mathcal{B}(\mathbb{C})$ to $\mathbb{R}$. We have shown that to $M$ there is associated a canonical element $\xi_{M} \in \mathcal{B}(\overline{\mathbb{Q}})$, independent of the choice of triangulation, with $\operatorname{Vol}(M)=D\left(\xi_{M}\right)$.

Algebraic $K$-theory. The discussion shows that if $F$ is a number field with $r_{2}=1$, then $\zeta_{F}(2)$ equals $\left|D_{F}\right|^{1 / 2} \pi^{2(n-1)} D(\xi)$ for some $\xi \in \mathcal{B}(\overline{\mathbb{Q}}) \otimes \mathbb{Q}$. However, much more is true: $\xi$ actually can be chosen to lie in the subgroup $\mathcal{B}(F) \otimes \mathbb{Q}$, and there is a corresponding result also for fields with $r_{2}>1$. To see this, the geometric method with triangulations is inadequate and one must use algebraic $K$-theory instead. To any ring $R$, there are associated algebraic $K$-groups $K_{0}(R)$, $K_{1}(R)=R^{\times}, K_{2}(R), K_{3}(R), \ldots$ The definition of these groups (which we will not use explicitly and hence do not describe) is complicated and highly non-constructive. However, in the case when $R=F$ is a number field, Borel [9] showed that the higher $K$-groups, modulo torsion, are free abelian groups of rank given by

$$
\operatorname{dim}_{\mathbb{Q}}\left(K_{n}(F) \otimes \mathbb{Q}\right)= \begin{cases}0, & n \geq 2 \text { even, } \\ n_{\mp}, & n=2 m-1>1, \mp=(-1)^{m-1},\end{cases}
$$

where $n_{+}=r_{1}+r_{2}$ and $n_{-}=r_{2}$ are the dimensions of the \pm -eigenspaces of complex conjugation on $F \otimes_{\mathbb{Q}} \mathbb{R} \cong \mathbb{R}^{r_{1}} \times \mathbb{C}^{r_{2}}$. Furthermore, for each $m>1$ there is a map reg ${ }_{m}: K_{2 m-1}(\mathbb{C}) \rightarrow \mathbb{R}$ which is $(-1)^{m-1}$-invariant w.r.t. complex conjugation on $\mathbb{C}$, and the composite map

$$
\operatorname{reg}_{m, F}: K_{2 m-1}(F) \longrightarrow\left(K_{2 m-1}(\mathbb{R})\right)^{r_{1}} \times\left(K_{2 m-1}(\mathbb{C})\right)^{r_{2}} \stackrel{\text { reg }_{m}}{\longrightarrow} \mathbb{R}^{n_{\mp}}
$$

maps $K_{2 m-1}(F) /\left(\right.$ torsion) isomorphically onto a cocompact lattice $\operatorname{Reg}_{m, F} \subset \mathbb{R}^{n_{\mp}}$ whose covolume is a rational multiple of $\left|D_{F}\right|^{1 / 2} \zeta_{F}(m) / \pi^{m n_{ \pm}}$.

In the case $n=3$, Bloch [5] gave a map between $K_{3}(F)$ and $\mathcal{B}(F)$, and it was shown later by Suslin [44] that in fact the two groups are canonically isomorphic (at least up to torsion; in this article we will consistently ignore torsion). Moreover, under this isomorphism the Borel regulator map on $K_{3}$ corresponds to the map $D$ on $\mathcal{B}(F)$, giving (the $\sim$ denotes an isomorphism up to finite kernel and cokernel)

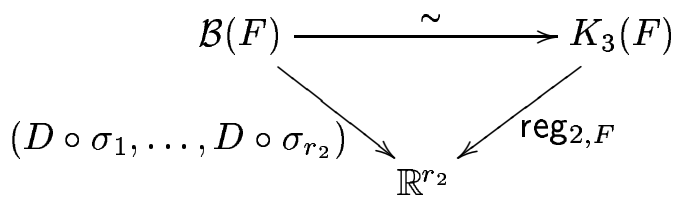

where $\sigma_{i}: F \hookrightarrow \mathbb{C}, i=1, \ldots, r_{2}$, denote the different complex embeddings (up to conjugation). Together with Borel's theorem, this yields in particular the 
Theorem. Let $F$ be a number field with $r_{1}$ real and $r_{2}$ complex places. Then we have:

(1) The group $\mathcal{B}(F)$ is finitely generated of rank $r_{2}$.

(2) Let $\xi_{1}, \ldots, \xi_{r_{2}}$ be a $\mathbb{Q}$-basis of $\mathcal{B}(F) \otimes \mathbb{Q}$ and $\sigma_{1}, \ldots, \sigma_{r_{2}}$ a set of complex embeddings (containing no pair of complex conjugate ones) of $F$ into $\mathbb{C}$. Then

$$
\zeta_{F}(2) \sim_{\mathbb{Q}^{\times}}\left|D_{F}\right|^{1 / 2} \cdot \pi^{2\left(r_{1}+r_{2}\right)} \cdot \operatorname{det}\left(D\left(\sigma_{i}\left(\xi_{j}\right)\right)_{1 \leq i, j \leq r_{2}}\right) .
$$

It is this statement which we wish to generalize to higher zeta-values.

\section{Formulation of the polylogarithm conjecture}

Higher polylogarithms. We now turn to the conjectures relating $K_{2 m-1}(F)$ and $\zeta_{F}(m)$ for higher values of $m$ to the $m^{\text {th }}$ polylogarithm function $L i_{m}(z)=\sum_{n>0} z^{n} / n^{m}$. The first step is to have at our disposal a single-valued version $\mathcal{L}_{m}$ of this function generalizing the function $D=: \mathcal{L}_{2}$. Ramakrishnan [38] showed that such a one-valued version exists, and explicit formulas were given in [50], [54], [53]. We will use the function

$$
\mathcal{L}_{m}(z)=\Re_{m}\left(\sum_{k=0}^{m-1} \frac{2^{k} B_{k}}{k !} \log ^{k}|z| L i_{m-k}(z)\right),
$$

where $\Re_{m}$ denotes $\Im$ or $\Re$ according as $m$ is even or odd and $B_{k}$ is the $k^{\text {th }}$ Bernoulli number. The series defining $L i_{r}$ converge exponentially for $|z|<1$ and $\mathcal{L}_{m}\left(z^{-1}\right)=(-1)^{m-1} \mathcal{L}_{m}(z)$, so we can easily compute $\mathcal{L}_{m}$ numerically to high accuracy. (A yet better method is indicated in [13], Proposition 1.) Extensive experiments suggested a definition of higher Bloch groups $\mathcal{B}_{m}(F)$. The idea behind the definition was that, by analogy, $\mathcal{B}_{m}(F)$ should be written as a quotient $\mathcal{A}_{m}(F) / \mathcal{C}_{m}(F)$ of a group $\mathcal{A}_{m}(F)$ of "allowable" elements (corresponding to the condition $\sum n_{i}\left(z_{i} \wedge\left(1-z_{i}\right)\right)=0$ in the case $\left.m=2\right)$ by a group $\mathcal{C}_{m}(F)$ of "concrete relations" coming from functional equations of the $m$-logarithm $\mathcal{L}_{m}$ (the relations in the case $m=2$ being induced by the five term relation).

Since $\mathcal{L}_{m}$ is $(-1)^{m-1}$-symmetric with respect to complex conjugation, the map $\mathcal{L}_{m}$ defines for any number field $F$ a map $\mathcal{L}_{m, F}: \mathcal{B}_{m}(F) \rightarrow \mathbb{R}^{n_{\mp}}$ and we expect the following diagram to commute:

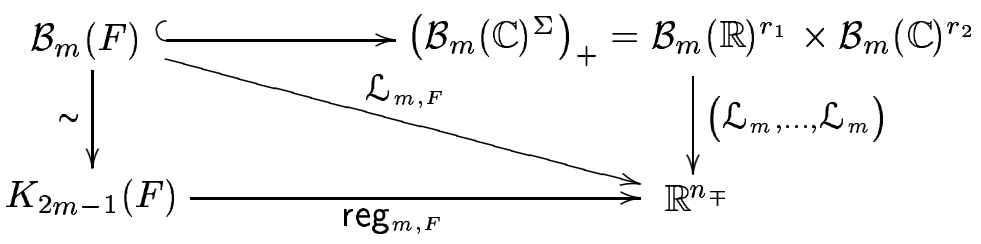

Here $\Sigma=\Sigma_{F}$ denotes the set of embeddings $F \hookrightarrow \mathbb{C}$ and ()$_{+}$the +-eigenspace with respect to the joint action of complex conjugation on $\Sigma_{F}$ and $\mathcal{B}_{m}(\mathbb{C})$, and $\mp$ is $(-1)^{m-1}$. If this picture is correct, then, by Borel's theorem, the evident analogues of the above-stated theorem hold: the vector space $\mathcal{B}_{m}(F) \otimes \mathbb{Q}$ has dimension $n_{\mp}$ over $\mathbb{Q}$, and if $\xi_{1}, \ldots, \xi_{n_{\mp}}$ is a basis of this vector space, then the determinant of the matrix $\mathcal{L}_{m}\left(\sigma_{i}\left(\xi_{j}\right)\right)_{i, j}$ is a non-zero rational multiple of $\left|D_{F}\right|^{1 / 2} \pi^{-m n_{ \pm}} \zeta_{F}(m)$.

Definition of the higher Bloch groups. The only question is how to define the groups $\mathcal{A}_{m}(F)$ and $\mathcal{C}_{m}(F)$. We describe the conjectural answer which was found by numerical experimentation and presented in [53]. For $m=2$ the definition of $\mathcal{A}_{m}(F)=\mathcal{A}(F)$ can be written more compactly as $\mathcal{A}_{2}(F)=\operatorname{ker} \beta_{2}$, where

$$
\beta_{2}: \mathbb{Z}[F] \rightarrow \bigwedge^{2} F^{\times}, \quad[z] \mapsto \begin{cases}z \wedge(1-z), & z \neq 1, \\ 0, & z=0,1 .\end{cases}
$$


The next simplest case occurs for $m=3$ and totally real $F$. Here we set $\mathcal{A}_{3}(F)=\operatorname{ker} \beta_{3}$ where $\beta_{3}$ is the map

$$
\beta_{3}: \mathbb{Z}[F] \rightarrow F^{\times} \otimes \bigwedge^{2} F^{\times}, \quad[z] \mapsto \begin{cases}z \otimes(z \wedge(1-z)), & z \neq 1, \\ 0, & z=0,1 .\end{cases}
$$

For instance, if $F=\mathbb{Q}$ then, as the reader can easily check, the elements

$$
\xi_{1}=\left[\frac{1}{2}\right], \quad \xi_{2}=2[3]-[-3], \quad \xi_{3}=6\left[\frac{2}{3}\right]+3\left[\frac{3}{4}\right]-3\left[\frac{1}{2}\right]-\left[\frac{8}{9}\right]-2\left[\frac{1}{3}\right]+\left[-\frac{1}{3}\right]
$$

are in $\mathcal{A}_{3}(\mathbb{Q})$, and one finds $\mathcal{L}_{3}\left(\xi_{1}\right)=\frac{7}{8} \zeta(3), \mathcal{L}_{3}\left(\xi_{2}\right)=\frac{13}{6} \zeta(3)$ and $\mathcal{L}_{3}\left(\xi_{3}\right)=\frac{67}{24} \zeta(3)$. Similarly, if $F=\mathbb{Q}(\sqrt{5})$, then the elements [1] and $\left[\frac{1+\sqrt{5}}{2}\right]$ belong to the kernel of $\beta_{3}$ and one finds

$$
\left(\begin{array}{cc}
\mathcal{L}_{3}(1) & \mathcal{L}_{3}(1) \\
\mathcal{L}_{3}\left(\frac{1+\sqrt{5}}{2}\right) & \mathcal{L}_{3}\left(\frac{1-\sqrt{5}}{2}\right)
\end{array}\right)=\left(\begin{array}{cc}
\zeta(3) & \zeta(3) \\
\frac{1}{10} \zeta(3)+\frac{25}{48} \sqrt{5} L\left(3, \chi_{5}\right) & \frac{1}{10} \zeta(3)-\frac{25}{48} L\left(3, \chi_{5}\right)
\end{array}\right)
$$

which has determinant $-\frac{25}{24} \sqrt{5} \zeta(3) L\left(3, \chi_{5}\right)=-\frac{25}{24} \sqrt{5} \zeta_{F}(3)$. (Here $L\left(s, \chi_{5}\right)$ denotes the $L$ series of the quadratic character $\chi_{5}$ associated to $\mathbb{Q}(\sqrt{5})$.) We can instead choose a basis like $\xi_{+}=\left[\frac{1+\sqrt{5}}{2}\right]+\left[\frac{1-\sqrt{5}}{2}\right]$ and $\xi_{-}=[2-\sqrt{5}]-[2+\sqrt{5}]+3\left(\left[\frac{1+\sqrt{5}}{2}\right]-\left[\frac{1-\sqrt{5}}{2}\right]\right)$, with $\xi_{+}$invariant and $\xi_{-}$anti-invariant under $\operatorname{Gal}(F / \mathbb{Q})$; then $\mathcal{L}_{3}\left(\xi_{+}\right)$is a rational multiple $\left(=\frac{1}{5}\right)$ of $\zeta(3)$ and $\mathcal{L}_{3}\left(\xi_{-}\right)$ a rational multiple $\left(=\frac{75}{32}\right)$ of $\sqrt{5} L\left(3, \chi_{5}\right)$, and this choice of basis diagonalizes the matrix.

For general number fields the condition $\xi \in \operatorname{ker}\left(\beta_{3}\right)$ turns out (experimentally) to be no longer sufficient to make $\xi$ land in a lattice with the desired properties, and in fact one needs a further condition, as was pointed out by Deligne. Observe first that if $\xi=\sum n_{i}\left[x_{i}\right] \in \operatorname{ker}\left(\beta_{3}\right)$, then for every homomorphism $\phi: F^{\times} \rightarrow \mathbb{Z}$ the element $\iota_{\phi}(\xi):=\sum n_{i} \phi\left(x_{i}\right)\left[x_{i}\right] \in \mathbb{Z}[F]$ belongs to $\mathcal{A}(F)=$ $\operatorname{ker}\left(\beta_{2}\right)$. The needed extra condition is that $\iota_{\phi}(\xi)$ in fact belongs to the subgroup $\mathcal{C}(F) \subset \mathcal{A}(F)$. This is not a restriction for $F$ totally real since then $\mathcal{A}(F) / \mathcal{C}(F) \cong K_{3}(F) \cong \mathbb{Z}^{r_{2}}=\{0\}$, but in the general case we obtain a group

$$
\mathcal{A}_{3}(F):=\left\{\xi \in \mathbb{Z}[F] \mid \iota_{\phi}(\xi) \in \mathcal{C}(F) \text { for all homomorphisms } \phi: F^{\times} \rightarrow \mathbb{Z}\right\}
$$

which is a proper subgroup of $\operatorname{ker}\left(\beta_{3}\right)$.

We now make the analogous definition for all $m$, setting

$$
\mathcal{A}_{m}(F):=\left\{\xi \in \mathbb{Z}[F] \mid \iota_{\phi}(\xi) \in \mathcal{C}_{m-1}(F) \quad \forall \phi \in \operatorname{Hom}\left(F^{\times}, \mathbb{Z}\right)\right\}
$$

This inductive definition requires knowing the previous " $\mathcal{C}$ "-group. The subgroup $\mathcal{C}_{m}(F)$ is supposed to be the group generated by the functional equations of $\mathcal{L}_{m}$, just as $\mathcal{C}(F)$ was generated by the five term relation. However, though one can give a formal definition based on this idea (see "Functional equations" below), it is not effective since the functional equations of the higher polylogarithms are not known explicitly. We therefore give a practical (i.e. numerically computable) definition of $\mathcal{C}_{m}(F)$ for number fields $F$ as

$$
\mathcal{C}_{m}(F):=\left\{\xi \in \mathcal{A}_{m}(F) \mid \mathcal{L}_{m}(\sigma(\xi))=0 \quad \forall \sigma \in \Sigma_{F}\right\}
$$

We can then formulate the polylogarithm conjecture for arbitrary $m$ : 
Conjecture. Let $F$ be a number field. Then the image of the map

$$
\mathcal{L}_{m, F}: \mathcal{A}_{m}(F) \longmapsto \mathbb{R}^{n_{\mp}}
$$

is commensurable with the Borel regulator lattice $\operatorname{Reg}_{m, F} \subset \mathbb{R}_{\mp}^{n_{\mp}}$. In particular,

(1) The group $\mathcal{B}_{m}(F):=\mathcal{A}_{m}(F) / \mathcal{C}_{m}(F)$ is finitely generated of rank $n_{\mp}$.

(2) Let $\xi_{j}\left(1 \leq j \leq n_{\mp}\right)$ be a $\mathbb{Q}$-basis of $\mathcal{B}_{m}(F) \otimes \mathbb{Q}$ and $\sigma_{i}\left(1 \leq i \leq n_{\mp}\right)$ run through $\Sigma$. Then

$$
\zeta_{F}(m) \sim_{\mathbb{Q}^{\times}}\left|D_{F}\right|^{1 / 2} \cdot \pi^{m n_{ \pm}} \cdot \operatorname{det}\left(\mathcal{L}_{m}\left(\sigma_{i}\left(\xi_{j}\right)\right)_{1 \leq i, j \leq n_{\mp}}\right) .
$$

We will discuss the evidence for this conjecture in $\S 3$. In the rest of this section we mention some supplementary aspects of its formulation.

Functional equations. The definition of $\mathcal{C}_{m}(F)$ which was given above leads to a formulation of the polylogarithm conjecture which can be tested numerically, but is rather artificial and works only for number fields. As already mentioned, $\mathcal{C}_{m}(F)$ should really be defined as "the group of all functional equations of the $m$-logarithm." We explain here how to do this. The new (and presumably equivalent) definition works for all fields and fits more naturally into a cohomological framework.

It is easy to see by induction that $\mathcal{A}_{m}(F)$ defined above is always a subgroup of the kernel of the map

$$
\beta_{m}=\beta_{m, F}: \mathbb{Z}[F] \rightarrow \operatorname{Sym}^{m-2} F^{\times} \otimes \bigwedge^{2} F^{\times}, \quad[z] \mapsto \begin{cases}z^{\otimes(m-2)} \otimes(z \wedge(1-z)), & z \neq 1, \\ 0, & z=0,1 .\end{cases}
$$

The "homotopic" definition of $\mathcal{C}_{m}(F)$ is then

$$
\mathcal{C}_{m}^{*}(F)=\left\{\xi(1)-\xi(0) \mid \xi(t) \in \operatorname{ker}\left(\beta_{m, F(t)}\right)\right\} .
$$

One can show [53, Prop. 1, p. 411] that an element $\xi(t) \in \mathbb{Z}[F(t)]$ is in the kernel of $\beta_{m, F(t)}$ if and only if the map $t \mapsto \mathcal{L}_{m}(\xi(t))$ is constant, so that the elements of $\mathcal{C}_{m}^{*}(F)$ really are the specializations to $F$ of all functional equations of $\mathcal{L}_{m}$. Clearly $\mathcal{C}_{m}^{*}(F) \subseteq \mathcal{C}_{m}(F)$, but the equality is known only for $m=2$.

It is highly non-trivial to find functional equations for $\mathcal{L}_{m}$. Kummer [30] gave special equations for $m=3,4,5$. Goncharov [23] found a functional equation in 3 variables for $\mathcal{L}_{3}$ which has a beautiful geometric interpretation in terms of configurations and is believed to generate $\mathcal{C}_{3}^{*}$ (i.e., to play the same role for the trilogarithm as does the five term relation for the dilogarithm). Examples of functional equations for higher $m$ (up to $m=7$ ) were given in [20]. A typical example for $m=6$ (cf. also [55]) is

$$
\sum_{(a, b) \in T} \frac{n_{a, b}}{\mu_{a, b}} \sum_{\substack{w \in W \\ z \in Z}} \mathcal{L}_{6}\left(\left(\frac{w}{z}\right)^{a}\left(\frac{1-w}{1-z}\right)^{b}\right)=\text { (independent of } y \text { ), }
$$

where $x$ and $y$ are independent variables,

$$
\begin{gathered}
T=\{(-2,3),(-2,1),(1,-2),(1,1),(1,0),(0,-1),(-1,1)\} \\
W=\left\{x(1-x),-\frac{(1-x)}{x^{2}},-\frac{x}{(1-x)^{2}}\right\}, \quad Z=\left\{y, 1-y, \frac{1}{1-y}, \frac{y}{y-1}, \frac{y-1}{y}, \frac{1}{y}\right\},
\end{gathered}
$$

and the coefficients $n_{a b}$ and $\mu_{a b}$ are defined by

$$
\sum_{(a, b) \in T} n_{a b}(a X+b Y)^{5}=0 \in \mathbb{Z}[X, Y], \quad \mu_{a b}=\log _{2}\left(1+a b+a^{2}+b^{2}\right) .
$$

For $m>7$, however, only the "trivial" (distribution and inversion) relations are known. 
Goncharov's complexes. The definition of $\mathcal{A}_{m}(F)$ can be written more elegantly as

$$
\mathcal{A}_{m}(F)=\operatorname{ker}\left(\mathbb{Z}[F] \stackrel{\iota}{\longrightarrow} F^{\times} \otimes \mathbb{Z}[F] / \mathcal{C}_{m-1}(F)\right)
$$

where $\iota: \mathbb{Z}[F] \rightarrow F^{\times} \otimes \mathbb{Z}[F]$ maps $[x]$ to $x \otimes[x]$ and $[0]$ to 0 . The group $\mathcal{B}_{m}(F)=\mathcal{A}_{m}(F) / \mathcal{C}_{m}(F)$ then becomes $\operatorname{ker}\left(\mathcal{G}_{m}(F) \stackrel{\partial}{\longrightarrow} F^{\times} \otimes \mathcal{G}_{m-1}(F)\right)$, where $\mathcal{G}_{m}(F)=\mathbb{Z}[F] / \mathcal{C}_{m}(F)$ and $\partial$ is the map induced by $\iota$. More generally, Goncharov [23] (using the notation $\mathcal{R}_{m}(F)$ for our $\mathcal{C}_{m}(F)$ and $\mathcal{B}_{m}(F)$ for our $\left.\mathcal{G}_{m}(F)\right)$ has defined for any field $F$ the complex

$\mathcal{G}_{m}(F) \stackrel{\partial}{\longrightarrow} F^{\times} \otimes \mathcal{G}_{m-1}(F) \stackrel{\partial}{\longrightarrow} \bigwedge^{2} F^{\times} \otimes \mathcal{G}_{m-2}(F) \stackrel{\partial}{\longrightarrow} \ldots \stackrel{\partial}{\longrightarrow} \bigwedge^{m-2} F^{\times} \otimes \mathcal{G}_{2}(F) \stackrel{\partial}{\longrightarrow} \bigwedge^{m} F^{\times}$

where $\partial$ maps $x_{1} \wedge \cdots \wedge x_{r} \otimes[x]$ to $x_{1} \wedge \cdots \wedge x_{r} \wedge x \otimes[x]$ (except in the last step, where $x_{1} \wedge \cdots \wedge x_{m-2} \otimes[x]$ is mapped to $\left.x_{1} \wedge \cdots \wedge x_{m-2} \wedge x \wedge(1-x)\right)$. The cohomology of this complex at the first point is then $\mathcal{B}_{m}(F)$, and Goncharov sharpens the conjecture of [53] to say that the cohomology groups of the complex give the graded pieces of a certain natural filtration on the algebraic $K$-groups, the Adams filtration. Of course, this only makes sense with the algebraic definition of $\mathcal{C}_{m}(F)$ in terms of functional equations, because the "practical" definition (6) applies to number fields only. Since the Adams filtration in the case of a number field is trivial after the first step, we can test Goncharov's conjecture in this case by testing the exactness of the above complex. The first non-trivial case is that

$$
\mathcal{G}_{4}(F) \stackrel{\partial}{\longrightarrow} F^{\times} \otimes \mathcal{G}_{3}(F) \stackrel{\partial}{\longrightarrow} \bigwedge^{2} F^{\times} \otimes \mathcal{G}_{2}(F)
$$

should be exact. This has been checked experimentally for $F=\mathbb{Q}$ in $[21]$, confirming the expected result.

Connection to hyperbolic volumes for $m>2$. The connection to hyperbolic volumes in the case $m=2$ does not simply extend to higher $m$. It is known by results of Schläfli that the formula for the volume of a hyperbolic $2 m$-simplex reduces to the formula for dimension $2 m-1$, so the volume of a 4-dimensional simplex can be expressed in terms of $D$. For hyperbolic simplices of dimension 5 and 6 it has been established that the volume can be expressed as a sum of values of trilogarithmic expressions (Böhm [8] (implicitly), Müller [36] (in principle, but with missing terms) and Kellerhals [29], cf. also Goncharov [24]). The volume of the 7-simplex, though, is not expressible in terms of $\mathrm{Li}_{4}$ alone (Goncharov, private communication). However, Goncharov conjectures [24] that the volume of any hyperbolic manifold of finite volume in dimension $2 m-1$ or $2 m$ should be expressible in terms of $\mathcal{L}_{m}$.

\section{Evidence for the conjecture}

Numerical evidence. The conjecture in the form given in $\S 2$ can be tested experimentally. Starting from $m=2$, we inductively want at each stage that (i) the images under $\mathcal{L}_{m, F}$ of the combinations found span a lattice, i.e. a discrete subgroup of $\mathbb{R}^{n} \mp$ of maximal rank, and (ii) the covolume is a simple multiple of $\zeta_{F}(m)$. The discreteness means that we can check numerically whether a given element of $\mathcal{A}_{m}$ belongs to $\mathcal{C}_{m}$ (its coordinates with respect to a $\mathbb{Z}$-basis of the lattice are integers, so can be checked to vanish by a low accuracy calculation), and this is just what we need to compute the next group $\mathcal{A}_{m+1}(F)$ and check that it again satisfies (i); and (ii) then provides a further test of the conjecture if the value of the zeta function is known.

Note that for a given element $\xi=\sum n_{i}\left[x_{i}\right] \in \mathbb{Z}\left[F^{\times}\right]$there are only finitely many conditions to be tested to determine whether $\xi$ belongs to $\mathcal{C}_{m}(F)$, since in (6) one can restrict to $\phi$ running over a basis of $\operatorname{Hom}(G, \mathbb{Z})$, where $G \subset F^{\times}$is the group generated by the $x_{i}$. More precisely, if we 
fix subgroups $G \subseteq G^{\prime} \subset F^{\times}$of ranks $r$ and $r^{\prime}$, respectively, and denote by $X$ the set of all $x \in G$ with $1-x \in G^{\prime}$, then the number of conditions for an element $\xi \in \mathbb{Z}[X]$ to lie in $\mathcal{A}_{m}(F)$ is

$$
\left(\begin{array}{c}
r+m-2 \\
m-1
\end{array}\right) r^{\prime}-\left(\begin{array}{c}
r+m-1 \\
m
\end{array}\right)+\sum_{k=1}^{m-2}\left(\begin{array}{c}
r+k-1 \\
k
\end{array}\right) n_{(-1)^{m-k-1}}
$$

and the number of conditions for $\xi$ to lie in $\mathcal{C}_{m}(F)$ is given by the same expression but with the sum starting at $k=0$. (The first two terms give the rank of $\operatorname{Sym}^{m-1}(X) \otimes X^{\prime} / \operatorname{Sym}^{m}(X)$ which is isomorphic to the target space of the map $\beta_{m}$ in $\operatorname{Sym}^{m-2}(X) \otimes \bigwedge^{2}\left(X^{\prime}\right)$.) This expression is polynomial in $r$ and linear in $r^{\prime}$, and a theorem of Erdős-Stewart-Tijdeman ([19]; cf. also [53, p. $425 \mathrm{ff}]$ or $[55$, p. $390 \mathrm{ff}]$ ) guarantees that the cardinality of $X$ grows much faster than this (at least for $F=\mathbb{Q}$, but experimentally for all number fields), so that in practice we can always find enough elements to generate the full lattice and test the conjecture.

This inductive process has been successfully carried out numerically in thousands of cases. Some examples will be described at the end of the section.

Compatibility with Galois descent. A consequence of the conjecture and the wellknown Galois descent for $K$-groups is the corresponding property for higher Bloch groups, i.e. $\mathcal{B}_{m}(F)=\mathcal{B}_{m}(E)^{G}$ for a Galois extension $E / F$ with Galois group $G$, and this property, which is highly non-trivial, can be checked in examples. For instance, consider $F=\mathbb{Q}(\sqrt{-7}) \subset E=\mathbb{Q}\left(\zeta_{7}\right)$ with $G=\mathbb{Z} / 3 \mathbb{Z}$. The element $\left[\zeta_{7}\right]$ trivially belongs to $\mathcal{A}_{m}(E)$, so its trace $\xi_{\text {cyc }}=\left[\zeta_{7}\right]+\left[\zeta_{7}^{2}\right]+\left[\zeta_{7}^{4}\right] \in$ $\mathcal{B}_{m}(E)^{G}$ should be equal to an element of $\mathcal{B}_{m}(F)$, which can be checked experimentally by computing its image in $\mathbb{R}$ under the map $\mathcal{L}_{m}$. For $m=2$ we can check explicitly that $\xi_{\text {cyc }}$ is equal modulo the five term relation to $\frac{4}{7} \xi_{7}$ with $\xi_{7}$ as in (1). Other examples will be given in $\S 5$.

Compatibility with Lichtenbaum's conjecture. Assuming both the conjecture above and Lichtenbaum's conjecture (which interprets the quotient of $\zeta$-value and regulator in terms of orders of $K$-groups) it is possible to not only verify inductively the above picture with computer experiments but it also allows to conjecturally predict orders of higher $K$-groups (cf. [11], [22], where the orders predicted agree with the few theoretical results known) and has moreover proved useful to detect a mistake in a proof concerning Lichtenbaum's conjecture.

Cyclotomic fields. Let $F=\mathbb{Q}\left(\zeta_{N}\right), N>2$. Here $n_{+}=n_{-}=\frac{1}{2} \phi(N)$ (Euler $\phi$-function). Take the $\xi_{j}$ to be $\left[\zeta_{N}^{l}\right](1 \leq l \leq N / 2,(l, N)=1)$. Clearly $\xi_{j}$ is in $\mathcal{A}_{m}(F)$ for all $m$ (since $\zeta_{N}^{l}$ is torsion in $F^{\times}$) and formula (7) is true in this case, as one sees by a standard calculation (cf. [53, p. 421ff]) using three facts:

i) The Dedekind zeta function $\zeta_{F}(s)$ is the product of $L(s, \chi)$ with $\chi$ running over all primitive Dirichlet characters of conductor dividing $N$,

ii) For a primitive character $\chi$ of conductor $f$, we have

$$
L(m, \chi)=\sum_{n=1}^{\infty} \frac{\chi(n)}{n^{m}}=\frac{1}{G(\bar{\chi})} \sum_{n=1}^{\infty} \sum_{r=1}^{f} \frac{\bar{\chi}(r) e^{2 \pi i r n / f}}{n^{m}}=\frac{1}{G(\bar{\chi})} \sum_{r=1}^{f} \bar{\chi}(r) L i_{m}\left(e^{2 \pi i r / f}\right),
$$

where $G(\bar{\chi})=\sum_{r=1}^{f} \bar{\chi}(r) e^{2 \pi i r / f}$ denotes the Gauss sum associated to $\bar{\chi}$.

iii) The Frobenius formula

$$
\operatorname{det}\left(t_{g h^{-1}}\right)_{g, h \in G}=\prod_{\chi \in \operatorname{Hom}(G, \mathbb{C} \times)} \sum_{g \in G} \chi(g) t_{g},
$$

valid for any finite abelian group $G$ and complex numbers $\left(t_{g}\right)_{g \in G}$. 
Abelian fields. If $F$ is abelian, then one obtains a partial result. By the Kronecker-Weber theorem, $F$ is a subfield of some cyclotomic field $E=\mathbb{Q}\left(\zeta_{N}\right)$. The Dedekind zeta function of $F$ still splits as $\prod_{\chi} L(s, \chi)$ where $\chi$ runs over a certain set of primitive characters (namely those which are trivial on $\left.\operatorname{Gal}(E / F) \subset \operatorname{Gal}(E / \mathbb{Q})=(\mathbb{Z} / N \mathbb{Z})^{\times}\right)$. The same calculation as for the cyclotomic case then gives us a lattice contained in $\mathcal{B}_{m}(E)^{\mathrm{Gal}(E / F)}$ of the expected rank $n_{\mp}(F)$ and which maps under $\mathcal{L}_{m, F}$ to a lattice in $\mathbb{R}^{n} \mp$ of the desired covolume.

General theoretical results. Finally, there are two main theoretical results in support of the conjecture. First of all, Beilinson and Deligne [4] and, independently, de Jeu [17] have shown that we can map the higher Bloch groups (in a somewhat different version than the one presented here) to the corresponding higher $K$-groups, and that the Borel regulator map in $K$-theory really is given by the polylogarithms. In particular, this suffices to show that there is a $\mathbb{Q}$-linear relation among the polylogarithms of any $n_{\mp}+1$ elements of the $m^{\text {th }}$ Bloch group. What is not known in general is the surjectivity, hence the expressibility of $\zeta_{F}(m)$ as a determinant of polylogarithms. In what is certainly the most important progress towards the general conjecture, Goncharov [23] proved this surjectivity for the case $m=3$. He also gave an explicit functional equation for the function $\mathcal{L}_{3}(z)$ with 22 terms in 3 variables which is believed to be the universal functional equation describing the subgroup $\mathcal{C}_{m}(F)$ in this case.

Examples. We illustrate the numerical procedure described at the beginning of the section. Example 1. The statement of the conjecture is non-trivial even for $F=\mathbb{Q}$, although the mere expressibility of the zeta-value in terms of polylogarithms is uninteresting in this case because $\zeta(m)$ is a rational multiple of $\pi^{m}$ for $m$ even and equals $\mathcal{L}_{m}(1)$ for $m$ odd. We illustrate a non-trivial verification up to the level of the 7-logarithm. Choose $G=\langle-1,2,3\rangle$ and $G^{\prime}=$ $\langle-1,2,3,5,7\rangle$. On the one hand, there are (at least) 29 rational numbers $x_{1}=\frac{1}{3}, x_{2}=-\frac{1}{3}$, $x_{3}=-\frac{1}{9}, \ldots x_{29}=-\frac{2}{243}$ satisfying $x \in G$ and $1-x \in G^{\prime}$. On the other hand, the number of conditions imposed on $\mathcal{A}_{7}(\mathbb{Q})$ by formula (8) with $r=2, r^{\prime}=4$, and $m=7$ is 28 , so we must get at least one element $\xi=\sum n_{i}\left[x_{i}\right]$ in $\mathcal{A}_{7}(\mathbb{Q})$. In fact, we find exactly one such combination (the coefficients, given explicitly on p. 386 of [55], are integers of up to 12 digits), and evaluating $\mathcal{L}_{7}(\xi)$ we find to very high accuracy a (huge) integer multiple of $\frac{1}{96} \zeta(7)$.

Example 2. A yet more impressive example is given in [13], where elements of the lattice $\mathcal{A}_{m}(F)$ are computed up to $m=16$ for a field $F$ of degree 10, namely the field generated by Lehmer's famous Salem number $\alpha$ (the algebraic number of conjecturally minimal height). Here the group $G$ is taken to be the group of rank 1 generated by -1 and $\alpha$ (i.e., this example is a so-called "ladder"), while $G^{\prime}$ is a group of small rank (generated by the units and a few small primes) which is chosen to contain many elements of the form $1 \pm \alpha^{i}$. At each stage, the (many) elements in $\mathcal{A}_{m}(F)$ found give vectors in $\mathbb{R}^{n_{\mp}}$ (with $n_{+}=6, n_{-}=4$ ) lying, within an accuracy of several hundred digits, in a lattice of the right rank. If we had missed a condition in the "correct" definition of $\mathcal{A}_{m}(F)$, it is very likely that it would have shown up before the level $m=16$. Very recently, Bailey and Broadhurst [1] extended this example one level higher, to the 17-logarithm.

Example 3. The third example, another ladder, is simpler than the two previous ones and will be used again later, so we give it in more detail. Let $F=\mathbb{Q}(\theta)$, where $\theta^{3}-\theta-1=0$, be the cubic field of discriminant -23 . Here $r_{1}=r_{2}=1$. We denote by $\sigma_{j}(0 \leq j \leq 2)$ the embeddings $F \hookrightarrow \mathbb{C}$ sending $\theta$ to $\theta_{0}=1.32471 \ldots$ (the real root), $\theta_{1}=\frac{\theta_{0}}{2}\left(-1+\frac{\sqrt{-23}}{2 \theta_{0}+3}\right)$, and $\theta_{2}=\bar{\theta}_{1}$, respectively. Let $G$ (=the group of units in $F$ ) be the group generated by -1 and $\theta$. The six elements

$$
x_{0}=1, x_{1}=\theta, x_{2}=-\theta, x_{3}=\theta^{3}, x_{4}=-\theta^{4}, x_{5}=\theta^{5}
$$

(and of course their inverses) have the property that both $x$ and $1-x$ belong to $G$. (We could also include $x_{6}=\theta^{2}$, but because of the "distribution relation" $2^{1-m} \mathcal{L}_{m}\left(\theta^{2}\right)=\mathcal{L}_{m}(\theta)+\mathcal{L}_{m}(-\theta)$ 
it would not add anything new.) Since $G$ has rank 1 , each $x_{i} \wedge\left(1-x_{i}\right)(1 \leq i \leq 5)$ vanishes (up to torsion), giving 6 elements $\alpha_{i}=\left[x_{i}\right](0 \leq i \leq 5)$ in $\mathcal{A}_{2}(F)$. The conjecture says that each $D\left(\sigma_{1}\left(\alpha_{i}\right)\right)=D\left(\sigma_{1}\left(x_{i}\right)\right)$ should be a rational multiple of $\pi^{-4} 23^{3 / 2} \zeta_{F}(2)$, and in fact we find

$$
D\left(\sigma_{1}\left(\alpha_{i}\right)\right)=\lambda_{i} \cdot \frac{3 \cdot 23^{3 / 2}}{8 \pi^{4}} \zeta_{F}(2), \quad\left(\lambda_{0}, \ldots, \lambda_{5}\right)=(0,1,-2,2,1,-1)
$$

(As already mentioned, polylogarithms can be computed rapidly using the formulas in [13]. We will indicate in $\S 5$ how to compute $\zeta_{F}(s)$ to high accuracy. It is easy to check directly that $\alpha_{i}$ equals $\lambda_{i} \alpha_{1}$ in the Bloch group.)

At the next stage, the condition for $\sum_{i=0}^{5} n_{i} \alpha_{i} \in \mathcal{B}_{3}(F)$ is that the element $\sum n_{i} \varepsilon_{i} \alpha_{i} \in \mathcal{A}_{2}(F)$ should map to 0 in $\mathcal{B}_{2}(F)$, i.e. $\sum n_{i} \varepsilon_{i} \lambda_{i}=0$, where $\varepsilon_{0}, \ldots, \varepsilon_{5}=0,1,1,3,4,5$ denote the exponents in $x_{i}= \pm \theta^{\varepsilon_{i}}$. This gives five non-trivial elements $\beta_{j}=\left[x_{j}\right]-\lambda_{j} \varepsilon_{j}\left[x_{1}\right]$ in $\mathcal{A}_{3}(F)$. We find

$$
\mathcal{L}_{3}\left(\sigma_{0}\left(\beta_{j}\right)\right)=\mu_{j} \cdot \frac{\zeta(3)}{3}+\nu_{j} \cdot \frac{23^{5 / 2}}{144 \pi^{3}} \frac{\zeta_{F}(3)}{\zeta(3)}, \quad \mathcal{L}_{3}\left(\sigma_{1}\left(\beta_{j}\right)\right)=\mu_{j} \cdot \frac{\zeta(3)}{3}-\nu_{j} \cdot \frac{23^{5 / 2}}{288 \pi^{3}} \frac{\zeta_{F}(3)}{\zeta(3)}
$$

with

$$
\left(\mu_{j}\right)_{j=0,2, \ldots, 5}=(3,0,3,2,1), \quad\left(\nu_{j}\right)_{j=0,2, \ldots, 5}=(0,3,-15,-13,13) .
$$

Thus, in accordance with the conjecture, the elements which we have found in $\mathcal{B}_{3}(F)$ lie in a 2-dimensional lattice $\mathbb{Z} \xi_{1}+\mathbb{Z} \xi_{2}$ with $\xi_{1}=\beta_{0}=[1], \xi_{2}=\beta_{5}-4 \beta_{2}=\left[x_{5}\right]-4\left[x_{2}\right]-3\left[x_{1}\right]$ and

$$
\left|\begin{array}{ll}
\mathcal{L}_{3}\left(\sigma_{0}\left(\xi_{1}\right)\right) & \mathcal{L}_{3}\left(\sigma_{0}\left(\xi_{2}\right)\right) \\
\mathcal{L}_{3}\left(\sigma_{1}\left(\xi_{1}\right)\right) & \mathcal{L}_{3}\left(\sigma_{1}\left(\xi_{2}\right)\right)
\end{array}\right|=\left|\begin{array}{cc}
\zeta(3) & \frac{\zeta(3)}{3}+\frac{23^{5 / 2}}{144 \pi^{3}} \frac{\zeta_{F}(3)}{\zeta(3)} \\
\zeta(3) & \frac{\zeta(3)}{3}-\frac{23^{5 / 2}}{288 \pi^{3}} \frac{\zeta F(3)}{\zeta(3)}
\end{array}\right| \underset{\mathbb{Q}^{\times}}{\sim} \pi^{-3} \sqrt{23} \zeta_{F}(3) .
$$

We now have three linear combinations $\left[x_{3}\right]+5\left[x_{2}\right]+4\left[x_{1}\right]-\left[x_{0}\right], 3\left[x_{4}\right]+13\left[x_{2}\right]+14\left[x_{1}\right]-2\left[x_{0}\right]$ and $\left[x_{5}\right]+\left[x_{4}\right]+\left[x_{1}\right]-\left[x_{0}\right]$ of the $\beta_{j}$ belonging to $\operatorname{ker}\left(\mathcal{L}_{3, F}\right)$. In order to deduce elements in $\mathcal{A}_{4}(F)$ from them, we first eliminate $\left[x_{0}\right]$ by taking appropriate linear combinations and then formally replace $\left[x_{i}\right]$ by $\frac{1}{\varepsilon_{i}}\left[x_{i}\right]$ ("pseudo-integration with respect to $\theta$ "). We are left with $\gamma_{4}=$ $9\left[x_{4}\right]-8\left[x_{3}\right]+36\left[x_{2}\right]+72\left[x_{1}\right]$ and $\gamma_{5}=\left[x_{5}\right]-4\left[x_{4}\right]+3\left[x_{3}\right]-46\left[x_{2}\right]-57\left[x_{1}\right]$, which are mapped by $\mathcal{L}_{4} \circ \sigma_{1}$ to $\frac{3}{128} \frac{23^{7 / 2}}{\pi^{4}} \frac{\zeta_{F}(4)}{\zeta(4)}$ and 0 , respectively. Finally, by "pseudo-integrating" $\gamma_{5}$, we obtain the combination $\delta_{5}=\frac{1}{5}\left[x_{5}\right]-\left[x_{4}\right]+\left[x_{3}\right]-46\left[x_{2}\right]-57\left[x_{1}\right] \in \mathcal{A}_{5}(F) \otimes \mathbb{Q}$, and the elements $\left[x_{0}\right]$ and $5\left(\delta_{5}-\left[x_{0}\right]\right)$ in $\mathcal{A}_{5}(F)$ map under $\mathcal{L}_{5, F}$ to $\zeta(5) \cdot(1,1)$ and $\frac{1}{128} \frac{23^{9 / 2}}{\pi^{5}} \frac{\zeta_{F}(5)}{\zeta(5)} \cdot(-2,1)$, respectively.

\section{The "enhanced" polylogarithm}

The enhanced regulator lattice. The regulator map $\operatorname{reg}_{m}: K_{2 m-1}(\mathbb{C}) \rightarrow \mathbb{R}$ is known to have a natural lift to $\mathbb{C} / \mathbb{Q}(m)$, and one can show (cf. [3]) that also the polylogarithm map $\mathcal{L}_{m}: \mathcal{B}_{m}(\mathbb{C}) \rightarrow \mathbb{R}$ enjoys this property (here we adopt the usual notation $\mathbb{Q}(m)=(2 \pi i)^{m} \mathbb{Q}$ ). We will call this lifted function, whose explicit definition will be described in this section, the enhanced polylogarithm and denote it by $\widehat{\mathcal{L}}_{m}$. If $F$ is a number field, then we can combine $\widehat{\mathcal{L}}_{m}$ as before with the embeddings $F \hookrightarrow \mathbb{C}$ to get a map $\widehat{\mathcal{L}}_{m, F}$ from $\mathcal{B}_{m}(F)$ to $(\mathbb{C} / \mathbb{Q}(m))_{+}^{\Sigma}$. Moreover, the construction of $\widehat{\mathcal{L}}_{m}$ is such that the indeterminacy on $\mathcal{B}_{m}(F)$ has bounded denominator, i.e. $N \cdot \widehat{\mathcal{L}}_{m, F}$ takes values in $(\mathbb{C} / \mathbb{Z}(m))_{+}^{\Sigma}$ for some integer $N=N(m, F)$. Note that $(\mathbb{C} / \mathbb{Z}(m))_{+}^{\Sigma}$ is topologically the product of the Euclidean space $\mathbb{R}^{n_{\mp}}$ with a torus $\left(\mathbb{R} /(2 \pi)^{m} \mathbb{Z}\right)^{n_{ \pm}}$. The inverse 
image under the projection $\mathbb{R}^{n}=\mathbb{C}_{+}^{\Sigma} \rightarrow(\mathbb{C} / \mathbb{Z}(m))_{+}^{\Sigma}$ of the image of $\mathcal{A}_{m}(F)$ under $N \cdot \widehat{\mathcal{L}}_{m, F}$ is then a cocompact lattice $\widehat{\mathcal{R}}_{m, F} \subset \mathbb{R}^{n}$;

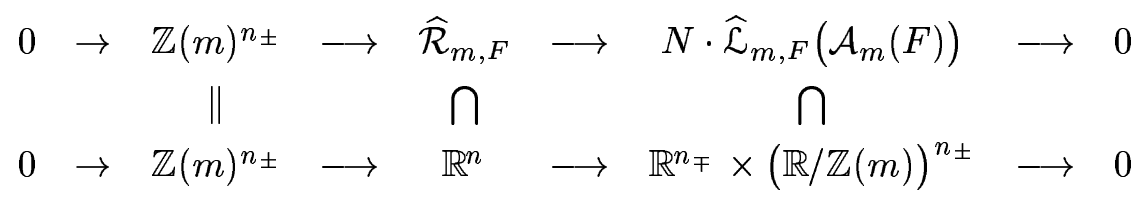

The regulator lattice $\mathcal{R}_{m, F}$ discussed in $\S 2$ had rank $n_{\mp}$. The new ("enhanced") lattice $\widehat{\mathcal{R}}_{m, F}$ has rank $n=n_{+}+n_{-}$and is (up to commensurability, as usual) the semi-direct product of $\mathcal{R}_{m, F}$ by the lattice $\mathbb{Z}(m)^{n_{ \pm}}$. In particular, its covolume contains no new information since it is, up to a rational factor, just $\pi^{m n_{ \pm}}$times the covolume of $\mathcal{R}_{m, F}$ : The fact that the lattice $\widehat{\mathcal{R}}_{m, F}$ is a semidirect product means that the torus $\mathbb{R}^{n} / \widehat{\mathcal{R}}_{m, F}$ is a torus bundle over $\mathbb{R}^{n} \mp / \mathcal{R}_{m, F}$ with fibre $(\mathbb{R} / \mathbb{Z}(m))^{n_{ \pm}}$, and the volumes multiply. Equivalently, the matrix representing $\widehat{\mathcal{R}}_{m, F}$ with respect to the standard basis of $\mathbb{R}^{n}$ has the form

$$
\left(\begin{array}{cc}
(2 \pi)^{m} \mathrm{I}_{n_{ \pm}} & * \\
0 & \text { matrix of } \mathcal{R}_{m, F}
\end{array}\right)
$$

so the determinant is just $(2 \pi)^{m n_{ \pm}}$times the previous determinant. The enhanced lattice is nevertheless of interest, for several reasons:

(1) It contains more information, as described by the off-diagonal block, whose elements are in general non-trivial (see example below).

(2) It gives a more elegant formulation of the main conjecture since the covolume of $\widehat{\mathcal{R}}_{m, F}$ is now a rational multiple of $\left|D_{F}\right|^{1 / 2} \zeta_{F}(m)$, without the extra factor $\pi^{m n_{ \pm}}$.

(3) One may sometimes have an independent construction of a candidate for the regulator lattice, and if this candidate also has an enhanced version then one can study the stronger conjecture that the enhanced lattices agree. We will see an example of this in $\S 7$.

Summarizing the above, we have

Using the $m^{\text {th }}$ enhanced polylogarithm on the Bloch group, we can associate to any number field $F$ of degree $n$ a regulator lattice $\widehat{\mathcal{R}}_{m, F} \subset \mathbb{R}^{n}$ for every $m \geq 2$ whose covolume is given (up to a rational number) by the value $\left|D_{F}\right|^{1 / 2} \zeta_{F}(m)$.

Lifting the dilogarithm. We describe explicitly how to construct the enhanced polylogarithm $\widehat{\mathcal{L}}_{m}$ in the case $m=2$. The first observation is that the ambiguity of the many-valued function

$$
F(u)=L i_{2}\left(e^{u}\right)-u L i_{1}\left(e^{u}\right), \quad(u \in \mathbb{C})
$$

is contained in $\mathbb{Z}(2)=(2 \pi i)^{2} \mathbb{Z}$. (Here $L i_{2}\left(e^{u}\right)$ and $L i_{1}\left(e^{u}\right)$ are to be defined by analytic continuation along the same path.) This is because the derivative of $F$ is the meromorphic function $u /\left(1-e^{-u}\right)$, which has only simple poles (at $\left.u=2 \pi i n, n \in \mathbb{Z}\right)$ with residue $(=2 \pi i n)$ in $2 \pi i \mathbb{Z}$. We can think of $F$ as a holomorphic function on $\mathbb{C}-(2 \pi i \mathbb{Z}-\{0\})$ with values in $\mathbb{C} / \mathbb{Z}(2)$. It satisfies the functional equations

$$
F(u)+F(-u)=\frac{\pi^{2}}{3}+\frac{u^{2}}{2}
$$

(Proof: both sides have derivative $u$ and equal $\frac{\pi^{2}}{3}$ at 0 ) and

$$
F(u+2 \pi i)-F(u)=2 \pi i \log \left(1-e^{u}\right) \in \mathbb{C} /(2 \pi i)^{2} \mathbb{Z}
$$


(Proof: both sides have derivative $\frac{2 \pi i}{1-e^{-u}}$ and vanish at $-\infty$ ). The well-definedness of $F$ modulo $\mathbb{Z}(2)$ says that the combination $L i_{2}(x)-\log (x) L i_{1}(x)$ becomes well-defined modulo $\mathbb{Z}(2)$ as soon as we choose a branch of $\log (x)$, i.e. a lift of $x$ to the abelian cover $\left\{u \in \mathbb{C} \mid e^{u} \neq 1\right\}$ of $\mathbb{C}-\{0,1\}$, and hence that $L i_{2}$ itself is well-defined modulo $\mathbb{Z}(2)$ on the maximal abelian cover $X=\left\{(u, v) \in \mathbb{C}^{2} \mid e^{u}+e^{v}=1\right\}$ of $\mathbb{C}-\{0,1\}\left(=\mathbb{P}^{1}(\mathbb{C})-\{0,1, \infty\}\right)$. Instead of $L i_{2}$ we use the function $\widehat{F}: X \rightarrow \mathbb{C} / \mathbb{Z}(2)$ defined by

$$
\widehat{F}(u, v)=F(u)-\frac{1}{2} u v .
$$

Its ambiguity with respect to changes of the logarithmic determinations $u$ and $v$ is given by

$$
\widehat{F}(u+2 \pi i r, v+2 \pi i s)=\widehat{F}(u, v)+\pi i(r v-s u)+2 \pi^{2} r s \quad(r, s \in \mathbb{Z}) .
$$

and its imaginary part is given by:

$$
\Im(\widehat{F}(u, v))=D\left(e^{u}\right)+\frac{1}{2} \Im(\bar{u} v),
$$

as one easily verifies.

If we now consider an element $\xi=\sum n_{j}\left[x_{j}\right]$ of $\operatorname{ker}\left(\beta_{2}\right)$, form the group $G=\left\langle x_{j}, 1-x_{j} \mid j\right\rangle$ and choose an arbitrary lifting of each $x_{j}$ to $X$, i.e. choose a logarithm $u_{j}$ of $x_{j}$ and a logarithm $v_{j}$ of $1-x_{j}$ for each $j$, then the complex values $u_{j}$ and $v_{j}$ all belong to the group $\widehat{G}=\left\{t \in \mathbb{C} \mid e^{t} \in G\right\}$, a free $\mathbb{Z}$-module of rank $=r k(G)+1$. (Here, as earlier, $G$ is the subgroup of $\mathbb{C}^{\times}$generated by all the $x_{j}$ and $\left.1-x_{j}.\right)$ The property $\sum n_{j}\left(x_{j} \wedge\left(1-x_{j}\right)\right)=0$ now becomes

$$
\sum n_{j}\left(u_{j} \wedge v_{j}\right)=2 \pi i \wedge A \in \wedge^{2}(\widehat{G})
$$

with $A \in \widehat{G}$ being well-defined modulo the subgroup $2 \pi i \mathbb{Z} \subset \widehat{G}$. We define the "enhanced dilogarithm" by

$$
\widehat{D}(\xi)=\sum n_{j} \widehat{F}\left(u_{j}, v_{j}\right)-\pi i A \in \mathbb{C} / 2 \pi^{2} \mathbb{Z} .
$$

Notice that we have taken the range of $\widehat{D}$ to be $\mathbb{C} / 2 \pi^{2} \mathbb{Z}$ rather than $\mathbb{C} / 4 \pi^{2} \mathbb{Z}$, because $A$ is defined only modulo $2 \pi i \mathbb{Z}$. It is easily verified that replacing $u_{j}$ and $v_{j}$ by $u_{j}+2 \pi i r_{j}$ and $v_{j}+2 \pi i s_{j}$ for some integers $r_{j}, s_{j}$ changes $A$ to $A+2 \pi i \sum n_{j}\left(r_{j} v_{j}-s_{j} u_{j}\right)$. Formula (12) then implies that $\widehat{D}(\xi)$ is independent of the chosen lifting $\left(u_{j}, v_{j}\right)$ of $x_{j}$, while formula $(13)$ gives $\Im(\widehat{D}(\xi))=D(\xi)$, since $\sum n_{j} \Im\left(\overline{u_{j}} v_{j}\right)=0$. The 5 -term relation for $D$ automatically remains valid for $\widehat{D}$. The function $\widehat{D}$ is then our desired lifting of $D: \mathcal{B}_{2}(\mathbb{C}) \rightarrow \mathbb{R}$ to $\mathbb{C} / 2 \pi^{2} \mathbb{Z}$.

If we look at an element $\xi=\sum n_{j}\left[x_{j}\right]$ with all $x_{j}$ real, then $\widehat{D}(\xi)$ is real by (13) and because $D(x)=0$ for $x$ real. Modulo $\pi^{2} / 2$ it is given by

$$
\widehat{D}(\xi)=\sum_{j} n_{j} R\left(x_{j}\right) \quad\left(\bmod \pi^{2} / 2\right)
$$

where $R: \mathbb{R} \rightarrow \mathbb{R}$ is the Rogers dilogarithm function, defined by the formulas

$$
R(x)= \begin{cases}L i_{2}+\frac{1}{2} \log (x) \log (1-x) & \text { if } 0<x<1 \\ \pi^{2} / 3-R(1 / x) & \text { if } x>1 \\ -\pi^{2} / 6+R(1 /(1-x)) & \text { if } x<0\end{cases}
$$


together with the values $R(0)=0, R(1)=\pi^{2} / 6$ dictated by continuity. We have $R(-\infty)=$ $-\pi^{2} / 6, R(+\infty)=\pi^{2} / 3$, so that $R$ gives a continuous function from $\mathbb{P}^{1}(\mathbb{R})=\mathbb{R} \cup\{\infty\}$ to $\mathbb{R} / \frac{1}{2} \pi^{2} \mathbb{Z}$. Thus, in the case of real arguments there is a version of the real part of the dilogarithm which is defined modulo $\pi^{2} / 2$ even for single arguments $x$ and we gain only a factor of 4 by considering elements of $\mathcal{A}_{2}(\mathbb{R})$, whereas in the complex case there is no lifting of individual dilogarithms at all and it is only on the subgroup $\mathcal{A}_{2}(\mathbb{C})$ of $\mathbb{Z}[\mathbb{C}$ that we get a lifting of the real part of the dilogarithm to the quotient of $\mathbb{R}$ by a lattice of rank 1 .

Example. We return to Example 3 of $\S 3$. There we gave six elements $\alpha_{i} \in \mathcal{A}_{2}(F)$, each of which is mapped to 0 under $\widehat{D} \circ \sigma_{0}$ and to a rational multiple of $\pi^{-4} \sqrt{23} \zeta_{F}(2)$ under $\widehat{D} \circ \sigma_{1}$. Let us consider the image of one of these elements, say $\alpha_{1}=[\theta]$, under the enhanced dilogarithm. (The other $\alpha_{j}$ give nothing new since $\alpha_{j}=\lambda_{j} \alpha_{1}$ in the Bloch group.) Let $t_{0}=\log \theta_{0}=0.281199 \ldots$, $t_{1}=\log \theta_{1}=-0.140599 \ldots+2.437734 \ldots i$. We have $1-\theta=-\theta^{-4}$, so for the real embedding we can take $u=t_{0}, v=-4 t_{0}+\pi i$, obtaining

$$
\widehat{D}\left(\sigma_{0}\left(\alpha_{1}\right)\right)=\widehat{F}\left(t_{0},-4 t_{0}+\pi i\right)+\frac{\pi i t_{0}}{2}=F\left(t_{0}\right)+2 t_{0}^{2}=R\left(\theta_{0}\right)=2.10466 \ldots,
$$

and similarly for the complex embedding

$$
\widehat{D}\left(\sigma_{1}\left(\alpha_{1}\right)\right)=\widehat{F}\left(t_{1},-4 t_{1}+\pi i\right)+\frac{\pi i t_{1}}{2}=-11.7444041862 \ldots+0.471353681388 \ldots \cdot i .
$$

Note that the original regulator lattice $\mathcal{R}_{2, F} \subset \mathbb{R}$ is the rank 1 group generated by the real value $D\left(\theta_{1}\right)=\Im\left(\widehat{D}\left(\theta_{1}\right)\right)$, whereas the new lattice $\widehat{\mathcal{R}}_{2, F} \subset \mathbb{R} \times \mathbb{C}$ is generated by the three vectors $\left(\widehat{D}\left(\theta_{0}\right), \widehat{D}\left(\theta_{1}\right)\right),\left(\pi^{2}, 0\right)$ and $\left(0, \pi^{2}\right)$, and (presumably) does not split over $\mathbb{Q}$ into the product of sublattices of $\mathbb{R}$ and $\mathbb{C}$, since the ratio of $\widehat{D}\left(\theta_{0}\right)$ and $\pi^{2}$ is (presumably) not a rational number. (We do, however, have the relation $\widehat{D}\left(\theta_{0}\right)+\widehat{D}\left(\theta_{1}\right)+\widehat{D}\left(\theta_{2}\right)=-13 \pi^{2} / 6$.)

Remark. We saw in $\S 1$ above that by triangulating a hyperbolic 3 -manifold $M$ one gets a well-defined element $\xi_{M} \in \mathcal{B}(\mathbb{C})$ whose image under $D$ is the value of $M$. The enhanced dilogarithm $\widehat{D}$ then gives us a finer invariant $\widehat{D}\left(\xi_{M}\right)$ whose imaginary part is the volume of $M$ and whose real part is a well-defined number in $\mathbb{C} / 2 \pi^{2} \mathbb{Z}$. According to a suggestion of Thurston, it should be (up to a normalizing factor) the Chern-Simons invariant of $M$. Cf. [47], [37], [51].

Lifting the polylogarithm. We begin by giving a different formula for the enhanced dilogarithm which is less precise than the above (it is defined modulo $\pi^{2} \mathbb{Q}$ only instead of $2 \pi^{2} \mathbb{Z}$ ) but is simpler and easier to generalize. For any complex number $U$ congruent to $\log x$ modulo $2 \pi i \mathbb{Q}$, we set

$$
\mathcal{F}(x, U)=L i_{2}(x)-U L i_{1}(x)=F(u)-(U-u) L i_{1}(x) \quad \in \mathbb{C} / \mathbb{Q}(2),
$$

where $u$ is any choice of logarithm of $x$. This is well-defined for a given choice of $u$ because $U-u \in 2 \pi i \mathbb{Q}$ and the ambiguity of $L i_{1}(x)$ lies in $2 \pi i \mathbb{Z}$, and it is independent of the choice of $u$ by (11). If now $\xi=\sum n_{j}\left[x_{j}\right]$ is any element of $\operatorname{ker}\left(\beta_{2}\right)$, and if $U_{j}$ and $V_{j}$ are any complex numbers satisfying

$$
U_{j} \equiv \log x_{j}(\bmod \mathbb{Q}(1)), \quad V_{j} \equiv \log \left(1-x_{j}\right)(\bmod \mathbb{Q}(1))
$$

(rather than actual choices of $\log x_{j}$ and $\log \left(1-x_{j}\right)$ like our previous $u_{j}, v_{j}$ ), and if we define $A \in \mathbb{C}^{\times} / \mathbb{Q}(1)$ by $\sum n_{j}\left(U_{j} \wedge V_{j}\right)=2 \pi i \wedge A$ as before, then one checks immediately that the expression

$$
\widehat{\mathcal{L}}_{2}(\xi):=\sum_{j} n_{j}\left(\mathcal{F}\left(x_{j}, U_{j}\right)-\frac{1}{2} U_{j} V_{j}\right)-\pi i A \in \mathbb{C} / \mathbb{Q}(2)
$$


is independent of the choices of $U_{j}$ and $V_{j}$ and is the reduction modulo $\mathbb{Q}(2)$ of the previously defined invariant $\widehat{D}(\xi) \in \mathbb{C} / \frac{1}{2} \mathbb{Z}(2)$. The advantage of the new construction is that there is a way of choosing $U_{j}$ and $V_{j}$ for which the number $A$ automatically vanishes, leading to a simpler definition. Let $\mathrm{L}$ be any homomorphism $\mathbb{C}^{\times} \rightarrow \mathbb{C}$ satisfying

$$
\mathrm{L}(x) \equiv \log (x)(\bmod \mathbb{Q}(1)) \quad \forall x \in \mathbb{C}^{\times},
$$

i.e. $\mathrm{L}$ is any lift

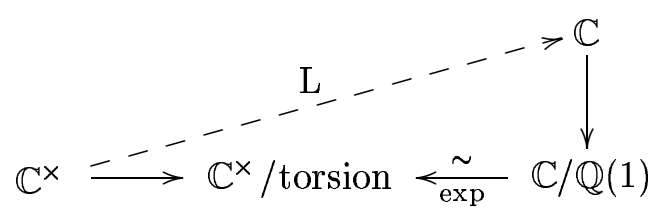

(Defining such a lift on all of $\mathbb{C}^{\times}$would require the axiom of choice, but we will only ever be using its restriction to a finitely generated $\operatorname{subgroup} G \subset \mathbb{C}^{\times}$- specifically, the group generated by the $x_{j}$ and $1-x_{j}$ for all $x_{j}$ occurring in some elements of the Bloch group of a number field - and then the lift can be defined simply by splitting $G$ as the sum of a free abelian and a finite group and choosing arbitrary logarithms of the generators of the free part.) Then with the choices $U_{j}=\mathrm{L}\left(x_{j}\right)$, $V_{j}=\mathrm{L}\left(1-x_{j}\right)$, we find immediately that the condition $\sum n_{j}\left(x_{j} \wedge\left(1-x_{j}\right)\right)=0 \in \bigwedge^{2}\left(\mathbb{C}^{\times}\right) \otimes \mathbb{Q}$ implies $A=0$, so we can define $\widehat{\mathcal{L}}_{2}(\xi)$ simply as

$$
\widehat{\mathcal{L}}_{2}(\xi):=\sum_{j} n_{j}\left(\mathcal{F}\left(x_{j}, \mathrm{~L}\left(x_{j}\right)\right)-\frac{1}{2} \mathrm{~L}\left(x_{j}\right) \mathrm{L}\left(1-x_{j}\right)\right) \in \mathbb{C} / \mathbb{Q}(2) \text {. }
$$

We can now generalize this to higher $m$. The function $F(u)$ is replaced by

$$
F_{m}(u)=\sum_{n=0}^{m-1} \frac{(-u)^{n}}{n !} L i_{m-n}\left(e^{u}\right)
$$

whose derivative $\frac{(-1)^{m}}{(m-1) !} \frac{u^{m-1}}{1-e^{-u}}$ has residues in $\frac{1}{(m-1) !} \mathbb{Z}(m-1)$ and which therefore is a well-defined holomorphic map from $\mathbb{C}$ to $\mathbb{C} / \frac{1}{(m-1) !} \mathbb{Z}(m-1)$. Its change under $u \mapsto u+2 \pi i$ is given by

$$
F_{m}(u+2 \pi i)=\sum_{n=0}^{m-1} \frac{(-2 \pi i)^{n}}{n !} F_{m-n}(u),
$$

and using this we can check that the expression

$$
\mathcal{F}_{m}(x, U)=\sum_{n=0}^{m-1} \frac{(\log x-U)^{n}}{n !} F_{m-n}(\log x) \in \mathbb{C} / \mathbb{Q}(m) \quad(U \in \mathbb{C}, U \equiv \log x(\bmod 2 \pi i \mathbb{Q})),
$$

is independent of the choice of $\log x$. In fact we can define $\mathcal{F}_{m}(x, U)$ directly, without choosing $\log x$ at all.

Proposition 1. Let $x \in \mathbb{C}-\{0,1\}$ and $U \in \mathbb{C}$ with $U \equiv \log (x)(\bmod \mathbb{Q}(1))$. Then the quantity

$$
\mathcal{F}_{m}(x, U)=\sum_{n=0}^{m-1} \frac{(-U)^{n}}{n !} L i_{m-n}(x)
$$


where all the polylogarithms $L i_{m-n}(x)$ are defined by analytic continuation along the same path, is well-defined. The effect of changing $U$ is given by the formula

$$
\mathcal{F}_{m}(x, U+\lambda)=\sum_{n=0}^{m-1} \frac{(-\lambda)^{n}}{n !} \mathcal{F}_{m-n}(x, U) \quad(\lambda \in \mathbb{Q}(1))
$$

Proof: The polylogarithm can be written as

$$
L i_{m}(x)=\frac{1}{(m-1) !} \int_{0}^{\infty} \frac{t^{m-1} d t}{x^{-1} e^{t}-1}
$$

(Proof: for $|x|<1$ expand $\frac{1}{x^{-1} e^{t}-1}$ in a geometric series and integrate term by term), so

$$
\mathcal{F}_{m}(x, U)=\frac{1}{(m-1) !} \int_{0}^{\infty} \frac{(t-U)^{m-1} d t}{x^{-1} e^{t}-1}
$$

The many-valuedness of this comes only from the choice of path of integration from 0 to $\infty$, and since the residues $\left\{(t-U)^{m-1} \mid e^{t}=x\right\}$ of the integrand are all in $\mathbb{Q}(m-1)$, the ambiguity which this produces is contained in $\mathbb{Q}(m)$, as asserted (and in fact with a controlled denominator: at most $N^{m-1}(m-1)$ ! if $x e^{-U}$ is an $N^{\text {th }}$ root of unity). The second statement of the proposition is an immediate consequence of the integral representation.

We now define the enhanced polylogarithm for $\xi=\sum n_{j}\left[x_{j}\right] \in \mathcal{A}_{m}(\mathbb{C})$ by

$$
\widehat{\mathcal{L}}_{m}(\xi)=\sum_{j} n_{j}\left(\mathcal{F}_{m}\left(x_{j}, \mathrm{~L}\left(x_{j}\right)\right)-\frac{(-1)^{m}}{m !} \mathrm{L}\left(x_{j}\right)^{m-1} \mathrm{~L}\left(1-x_{j}\right)\right)
$$

where $\mathrm{L}$ as before is any lift to $\mathbb{C}$ of the logarithm map $\mathbb{C}^{\times} \rightarrow \mathbb{C} / \mathbb{Q}(1)$.

Proposition 2. Let $\xi \in \mathcal{A}_{m}(\mathbb{C}), m \geq 2$. Then:

(i) The value of $\mathcal{L}_{m}(\xi)$ is independent of the choice of $\mathrm{L}$.

(ii) $\Re_{m}\left(\widehat{\mathcal{L}}_{m}(\xi)\right)=\mathcal{L}_{m}(\xi)$.

(iii) $\mathcal{L}_{m}(\xi)=0$ if $\xi \in \mathcal{C}_{m}(\mathbb{C})$.

Proof: We prove only (i), the ideas for (ii) and (iii) being similar. We must show that the number $\widehat{\mathcal{L}}_{m}^{*}(\xi)$ obtained by replacing $\mathrm{L}$ by $\mathrm{L}^{*}=\mathrm{L}+\lambda$, where $\lambda$ is a homomorphism from $\mathbb{C}^{\times}$to $\mathbb{Q}(1)$, is equal to $\widehat{\mathcal{L}}_{m}(\xi)$ (modulo $\mathbb{Q}(m)$ ) for $\xi \in \mathcal{A}_{m}(\mathbb{C})$. We have

$$
\begin{aligned}
\mathrm{L}^{*}(x)^{m-1} \mathrm{~L}^{*}(1-x)-\mathrm{L}(x)^{m-1} \mathrm{~L}(1-x) & \\
\equiv & \sum_{n=0}^{m-2}\left(\begin{array}{c}
m-1 \\
n
\end{array}\right) \mathrm{L}(x)^{n} \lambda(x)^{m-n-1} \mathrm{~L}(1-x)+\sum_{n=1}^{m-1}\left(\begin{array}{c}
m-1 \\
n
\end{array}\right) \mathrm{L}(x)^{n} \lambda(x)^{m-n-1} \lambda(1-x) \\
= & \sum_{n=1}^{m-1}\left(\begin{array}{c}
m-1 \\
n
\end{array}\right) \mathrm{L}(x)^{n-1} \lambda(x)^{m-n-1}(\mathrm{~L}(x) \lambda(1-x)-\mathrm{L}(1-x) \lambda(x)) \\
& \quad+\sum_{n=1}^{m-1}\left(\begin{array}{c}
m \\
n
\end{array}\right) \mathrm{L}(x)^{n-1} \lambda(x)^{m-n} \mathrm{~L}(1-x)
\end{aligned}
$$


where $\equiv$ denotes congruence modulo $\mathbb{Q}(m)$ and in the second line we have used the identity $\left(\begin{array}{c}m-1 \\ n-1\end{array}\right)+\left(\begin{array}{c}m-1 \\ n\end{array}\right)=\left(\begin{array}{c}m \\ n\end{array}\right)$. From this and (14) it follows that

$$
\begin{aligned}
\widehat{\mathcal{L}}_{m}^{*}(\xi) & -\widehat{\mathcal{L}}_{m}(\xi)=\sum_{n=1}^{m-2} \frac{(-1)^{n}}{n !} \widehat{\mathcal{L}}_{m-n}\left(\sum_{j} n_{j} \lambda\left(x_{j}\right)^{n}\left[x_{j}\right]\right) \\
& -\frac{(-1)^{m}}{m !} \sum_{n=1}^{m-1}\left(\begin{array}{c}
m-1 \\
n
\end{array}\right) \sum_{j} n_{j} \mathrm{~L}\left(x_{j}\right)^{n-1} \lambda\left(x_{j}\right)^{m-n-1}\left(\mathrm{~L}\left(x_{j}\right) \lambda\left(1-x_{j}\right)-\mathrm{L}\left(1-x_{j}\right) \lambda\left(x_{j}\right)\right) .
\end{aligned}
$$

The first sum vanishes by inductive use of (iii) because $\sum n_{j} \lambda\left(x_{j}\right)^{n}\left[x_{j}\right] \in \mathcal{C}_{m-n}(\mathbb{C})$, and the second because $\sum n_{j}\left[x_{j}\right] \in \operatorname{ker} \beta_{m}$.

The content of the proposition, and of this whole section, can be summarized by the following diagram:

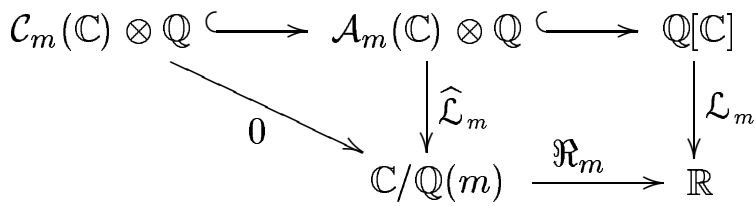

Example. Again, we take up Example 3 of $\S 3$ and compute the enhanced trilogarithm for the elements $\beta_{j}$ of $\mathcal{B}_{3}(F)(j=0,2, \ldots, 5)$. We need only consider $\sigma_{1}$ since the imaginary part of $\widehat{\mathcal{L}}_{3}(\xi)$ changes sign under $\xi \mapsto \bar{\xi}$. Choosing $\mathrm{L}\left(\theta_{1}\right)=\log \left(\theta_{1}\right)=-0.1405 \ldots+2.4377 \ldots i$, we obtain

$$
\widehat{\mathcal{L}}_{3}\left(\sigma_{1}\left(\beta_{j}\right)\right)=\mu_{j} \frac{\zeta(3)}{3}+\nu_{j}\left(-\frac{23^{5 / 2}}{288 \pi^{2}} \frac{\zeta_{F}(3)}{\zeta(3)}+f \cdot i\right)+\omega_{j} \cdot \frac{(2 \pi i)^{3}}{24}
$$

for a certain real number $f=15.13531974616481725314880543 \ldots$, where the integers $\mu_{j}$ and $\nu_{j}$ are as in Example 3 of $\S 3$ and $\left(\omega_{j}\right)_{j=0,2, \ldots, 5}=(0,0,3,19,23)$. If we choose instead $\mathrm{L}^{*}\left(\theta_{1}\right)=$ $\mathrm{L}\left(\theta_{1}\right)-i \pi$, then we find the same formula with $\left(\omega_{j}\right)_{j}=\frac{1}{2}(0,12,-15,-14,11)$. The number $f$ can be considered as an "imaginary part" of $-\frac{23^{5 / 2}}{288 \pi^{2}} \frac{\zeta_{F}(3)}{\zeta(3)}$, defined only modulo $\pi^{3} \mathbb{Q}$ (or more precisely, $\frac{\pi^{3}}{3} \mathbb{Z}$ ). We will give an interpretation of this somewhat cryptic statement in the following chapter. 


\section{PART II. A HIGHER KRONECKER LIMIT FORMULA FOR IMAGINARY QUADRATIC FIELDS}

\section{A conjectural higher Kronecker limit formula}

Let $E / F$ be an extension of number fields, which we may assume to be Galois. Then the zeta function of $E$ can be written as the product of so-called Artin $L$-series $L_{F}(s, \rho)$ associated to an irreducible representation $\rho$ of the Galois group $G$. In particular, the residue $\operatorname{res}_{s=1} \zeta_{E}(s)$ is a product of $\operatorname{res}_{s=1} \zeta_{F}(s)$ and special values $L_{F}(1, \rho)$. On the other hand, the residue is the covolume of the group of units $\mathcal{O}_{E}^{\times}$on which $G$ acts, which implies that $\mathcal{O}_{E}^{\times}$(at least after tensoring with $\mathbb{C}$ or $\overline{\mathbb{Q}}$ ) decomposes as a direct sum of irreducible representations of $G$ and consequently that the regulator decomposes into a corresponding product of factors. Comparing these two facts, one is led to the famous

Conjecture (Stark). The value $L_{F}(1, \rho)$ is expressible as a simple multiple (a power of $\pi$ times an algebraic number) of the determinant of a matrix whose entries are logarithms of units in $E$.

This conjecture which was formulated in far greater precision by Stark [43] is totally open except in very special cases, but one can nevertheless generalize it to higher special values. The number $\zeta_{E}(m)$ is both a product of special values of $L_{F}(m, \rho)$ and, by Borel's theorem, the covolume of the regulator lattice $\operatorname{Reg}_{m, E}$ on which $G$ acts. Therefore it is natural to guess that each special value $L_{F}(m, \rho)$ is the volume of one of the lattices into which the $G$-action splits $\operatorname{Reg}_{m, E}$. This conjecture, which was made by Gross in 1974 [27], combined with the conjectural description of the Borel regulator map in terms of polylogarithms, gives the

Strengthened polylogarithm conjecture. The value at $s=m$ of any Artin L-function $L_{F}(s, \rho)$ is a simple multiple of the determinant of a matrix whose entries are polylogarithms evaluated at elements in the higher Bloch group $\mathcal{B}_{m}(E)$.

Again, one can easily make this a little more precise, and could probably make a very detailed analysis along the lines of the one given in [45] for $m=1$ if one wished, but this seems pointless as long as even the case $m=1$ remains unapproachable.

A particularly interesting special case arises if we concentrate on abelian extensions of an imaginary quadratic field $K$, where we can formulate a much more precise statement which in the case $m=1$ reduces to a famous classical theorem. For simplicity we restrict to the Hilbert class field $H$ of $K$. We first observe that the zeta function $\zeta_{H}(s)$ of $H$ can be expressed as the determinant of a matrix whose entries are the partial zeta functions of the quadratic field. This follows easily from the well-known decomposition of $\zeta_{H}$ into $L$-series associated to ideal class characters $\chi$ :

$$
\begin{aligned}
\zeta_{H}(s) & =\prod_{\chi: \mathcal{C} \ell_{K} \rightarrow \mathbb{C}^{\times}} L_{K}(s, \chi)=\prod_{\chi}\left(\sum_{\mathcal{A} \in \mathcal{C} \ell_{K}} \chi(\mathcal{A}) \zeta_{K, \mathcal{A}}(s)\right) \\
& =\operatorname{det}\left(\left(\zeta_{K, \mathcal{A B}}-1(s)\right)_{\mathcal{A}, \mathcal{B} \in \mathcal{C} \ell_{K}}\right) .
\end{aligned}
$$

(Here $\mathcal{C} \ell_{K}$ denotes the ideal class group, $L_{K}(s, \chi)=\sum_{\mathfrak{a}} \chi(\mathfrak{a}) N(\mathfrak{a})^{-s}$ the associated $L$-series and $\zeta_{K, \mathcal{A}}(s)=\sum_{\mathfrak{a} \in \mathcal{A}} N(\mathfrak{a})^{-s}$ the partial zeta function associated to the ideal class $\mathcal{A}$. The final identity is a special case of the theorem of Frobenius about group determinants and can be proved easily since conjugation by the matrix $(\chi(\mathcal{A}))_{\chi, \mathcal{A}}$ diagonalizes the matrix of partial zeta 
functions.) But by the conjecture stated in $\S 2$ we expect $\zeta_{H}(m)(m=2,3,4, \ldots)$ to be a multiple of the determinant of an $h \times h$-matrix, where $h=\# \mathcal{C} \ell_{K}=[H: K]=n_{ \pm}(H)$, whose entries are polylogarithms evaluated at arguments in $H$. (If $H$ were an arbitrary abelian extension of $K$ instead of the maximal unramified one, then $\mathcal{C} \ell_{K}$ would be replaced by a ray class group, but the analysis would be otherwise identical.) The case $s=1$ where the values of the partial zeta functions can be written in terms of logarithms of units in $F$ is a consequence of Kronecker's limit formula and the theory of complex multiplication, and the construction of units in the Hilbert class field from special values of modular forms constitutes one of the high points of $19^{\text {th }}$ century number theory.

It is therefore natural to guess that in fact the individual entries agree and that each partial zeta-value $\zeta_{K, \mathcal{A}}(\mathrm{m})$ is a linear combination of polylogarithm values, and in fact, numerical examples suggested its statement. We consider $K$ as embedded into $\mathbb{C}$. Then there is a distinguished embedding of $H$ into $\mathbb{C}$ as $K\left(j\left(z_{1}\right)\right)$ where $j: \mathbb{H} / \mathrm{SL}(2, \mathbb{Z}) \rightarrow \mathbb{C}$ is the classical modular invariant and $z_{1}=\frac{D+\sqrt{D}}{2} \in \mathbb{H}$, the upper half plane. The other embeddings of $H$ into $\mathbb{C}$ are obtained from this one by conjugating it with the elements of $\operatorname{Gal}(H / K)$ and with complex conjugation. Moreover, by class field theory $\operatorname{Gal}(H / K)$ is isomorphic to $\mathcal{C} \ell_{K}$ via the Artin symbol. A fairly precise formulation of the conjecture indicated above is then:

Conjecture. Let $K$ be an imaginary quadratic number field and $m>1$. Then there is an element $\xi=\xi_{m, K} \in \mathcal{B}_{m}(H) \otimes \mathbb{Q}$, where $H$ is the Hilbert class field of $K$, such that

$$
\zeta_{K, \mathcal{A}}(m)=2 \frac{(-1)^{\left[\frac{m-1}{2}\right]}(2 \pi)^{m}}{(m-1) !}\left|D_{K}\right|^{\frac{1}{2}-m} \mathcal{L}_{m}\left(\sigma_{\mathcal{A}}(\xi)\right)
$$

for every ideal class $\mathcal{A}$ of $K$, where $\sigma_{\mathcal{A}} \in \operatorname{Gal}(H / K)$ is the Artin symbol associated to $\mathcal{A}$.

For $m=1$ the statement must be modified because each $\zeta_{F}(m)$ has a simple pole, but $\zeta_{H}(s) / \zeta_{K}(s)=\prod_{\chi \neq 1} L_{m}(s, \chi)$ is finite at $s=1$ and can be expressed as a determinant of values $\left.\left(\zeta_{K, \mathcal{A}}(s)-\zeta_{K, \mathcal{B}}(s)\right)\right|_{s=1}$ where $\mathcal{A}$ and $\mathcal{B}$ denote ideal classes in $K$, and the Kronecker limit formula expresses each of these differences as the logarithm of a certain element in $H$ defined by complex multiplication theory.

Remarks. 1. $\xi_{m, K}$ is unique if it exists (assuming the general polylogarithm conjecture), since by specifying $\mathcal{L}_{m}(\sigma(\xi))$ for all $\sigma \in \operatorname{Gal}(H / K)$ we have specified the image of $\xi$ under the full map $\mathcal{L}_{m, H}: \mathcal{A}_{m}(H) \rightarrow \mathbb{R}^{h}$, and the polylogarithm conjecture says that this map is injective (up to torsion). It also follows that $c\left(\xi_{m, K}\right)=(-1)^{m-1} \xi_{m, K}$, where $c \in \operatorname{Gal}(H / \mathbb{Q})$ denotes complex conjugation.

2. The partial zeta function $\zeta_{K, \mathcal{A}}(s)$ in the case of an imaginary quadratic field $K$ can also be written as an Epstein zeta function: the norms of the integral ideals in $\mathcal{A}$ are just the values attained by some quadratic form $Q(x, y)=a x^{2}+b x y+c y^{2}(a, b \in \mathbb{Z})$ with discriminant $b^{2}-4 a c=$ $D_{K}$, so

$$
\zeta_{K, \mathcal{A}}(s)=\zeta_{Q}(s)=\frac{1}{2} \sum_{x, y}^{\prime} \frac{1}{Q(x, y)^{s}} .
$$

(Here the prime means that $(x, y)=(0,0)$ is to be omitted and the factor $\frac{1}{2}$ takes care of the automorphism $(x, y) \mapsto(-x,-y)$. If $K=\mathbb{Q}(i)$ or $K=\mathbb{Q}(\sqrt{-3})$, it must be replaced by $\frac{1}{4}$ or $\frac{1}{6}$, but we will ignore these cases since the conjecture is only interesting if $h>1$.) Thus the conjecture says in particular:

Let $Q$ be any positive definite binary quadratic form with rational coefficients and let $m \geq 2$. Then $|\operatorname{disc}(Q)|^{-1 / 2} \pi^{-m} \zeta_{Q}(m)$ is a $\mathbb{Q}$-linear combination of values of $\mathcal{L}_{m}(x)$ with $x$ algebraic. 
3. The partial zeta function can also be written as a non-holomorphic Eisenstein series: if $Q(m, n)=a m^{2}+b m n+c n^{2}$ is a form in the class associated to $\mathcal{A}$, and we set $z_{Q}=\frac{b+\sqrt{D_{K}}}{2 a} \in \mathbb{H}$, then $Q(m, n)=\left|D_{K} / 4\right|^{1 / 2}\left|m z_{Q}+n\right|^{2} / \Im\left(z_{Q}\right)$, so

$$
\zeta_{Q}(s)=\left|D_{K} / 4\right|^{-s / 2} E\left(z_{Q}, s\right)
$$

where

$$
E(z, s)=\frac{1}{2} \sum_{m, n \in \mathbb{Z}}^{\prime} \frac{\Im(z)^{s}}{|m z+n|^{2 s}} \quad(z \in \mathbb{H}, \Re(s)>1),
$$

is the non-holomorphic Eisenstein series of weight 0. This is useful because the Fourier expansion of $E(z, s)$ is explicitly known and converges with exponential rapidity (at least like $\sum e^{-n \pi \sqrt{3}}$ ), so that we can compute special values of partial zeta functions very easily.

Examples. 1. Let $K=\mathbb{Q}(\sqrt{-23})$. The class number is 3 and the Hilbert class field is $H=K(\theta)=K \cdot F$, where $F=\mathbb{Q}(\theta)$ with $\theta^{3}-\theta-1=0$ is the field considered in Example 3 of $\S 3$. The class group $\mathcal{C} \ell_{K}$ consists of the classes $\mathcal{A}_{0}=\{(\lambda)\}$ (the principal class), $\mathcal{A}_{1}=\{(\lambda) \wp\}$ and $\mathcal{A}_{-1}=\{(\lambda) \bar{\wp}\}$ where $\wp=\left(2, \frac{1+\sqrt{-23}}{2}\right)$ is one of the prime ideals dividing 2 . The corresponding zeta functions $\zeta_{j}(s)=\zeta_{K, \mathcal{A}_{j}}(s)(j \in \mathbb{Z} / 3 \mathbb{Z})$ are given by

$$
\zeta_{0}(s)=\frac{1}{2} \sum_{m, n}^{\prime} \frac{1}{\left(m^{2}+m n+6 n^{2}\right)^{s}}, \quad \zeta_{ \pm 1}(s)=\frac{1}{2} \sum_{m, n}^{\prime} \frac{1}{\left(2 m^{2}+m n+3 n^{2}\right)^{s}} .
$$

These partial zeta functions are related to the Dedekind zeta functions of $K$ and $F$ by

$$
\zeta_{0}(s)+2 \zeta_{1}(s)=\zeta_{K}(s), \quad \zeta_{0}(s)-\zeta_{1}(s)=\frac{\zeta_{F}(s)}{\zeta(s)}
$$

so by formulas (2) and (9) with $i=1$, their values at $s=2$ are expressible in terms of dilogarithms as

$$
\zeta_{0}(2)=\frac{4 \pi^{2}}{9 \cdot 23^{3 / 2}}\left(D\left(\xi_{23}\right)+24 D\left(\theta_{1}\right)\right), \quad \zeta_{1}(2)=\frac{4 \pi^{2}}{9 \cdot 23^{3 / 2}}\left(D\left(\xi_{23}\right)-12 D\left(\theta_{1}\right)\right),
$$

where we have labelled the conjugates of $\theta$ as $\theta_{j}(j \in \mathbb{Z} / 3 \mathbb{Z})$ as in $\S 3$. We can check these formulas numerically since $\zeta_{0}(2)$ and $\zeta_{1}(2)$ can be computed rapidly using (16) and (24) below:

$$
\begin{aligned}
\zeta_{0}(2) & =\frac{\pi^{4}}{90}+\frac{8 \pi}{23 \sqrt{23}}\left(\frac{1}{2} \zeta(3)+\sum_{n=1}^{\infty}(-1)^{n} \sigma_{-3}(n)(1+\pi n \sqrt{23}) e^{-\pi n \sqrt{23}}\right) \\
& =1.2192662262804613691002600581331744360615022454119 \ldots, \\
\zeta_{1}(2) & =\frac{\pi^{4}}{360}+\frac{16 \pi}{23 \sqrt{23}}\left(\frac{1}{2} \zeta(3)+\sum_{n=1}^{\infty}(-1)^{n} \sigma_{-3}(2 n)(1+\pi n \sqrt{23}) e^{-\pi n \sqrt{23}}\right) \\
& =0.5444665316326198108031691850861304122679869701278 \ldots,
\end{aligned}
$$

where $\sigma_{-3}(n)=\sum_{d \mid n} d^{-3}$. Equation (17) verifies the conjecture for this field and $m=2$ with

$$
\xi_{2, K}=\frac{1}{18}\left(\left[\xi_{23}\right]+12[\sigma(\theta)]-12\left[\sigma^{2}(\theta)\right]\right)
$$

where $\sigma$ is a generator of the (cyclic) Galois group $\mathrm{Gal}(H / K)$. Of course this above representation as a sum of dilogarithms is not unique. We give a different expression which is more homogeneous 
with respect to the action of the Galois group. The unit group of $H$ is generated by $\theta_{0}, \theta_{1}$ and $\theta_{2}$ with the relation $\theta_{0} \theta_{1} \theta_{2}=1$. Then the elements

$$
\xi_{H,-23}^{(j)}=8\left[-\theta_{j}^{4} \theta_{j+1}^{2}\right]-5\left[-\theta_{j}^{4} \theta_{j+1}^{3}\right]+21\left[-\theta_{j}^{2} \theta_{j+1}\right] \quad(j \in \mathbb{Z} / 3 \mathbb{Z}),
$$

satisfy

$$
D\left(\xi_{H,-23}^{(j)}\right)=-\frac{3 \cdot 23^{3 / 2}}{2 \pi^{2}} \zeta_{j}(2)
$$

and are cyclically permuted by $\operatorname{Gal}(H / K)$, so that the element $\xi_{2, K}:=-\frac{1}{12} \xi_{H,-23}$ has the property required by the conjecture.

2. For other $K=\mathbb{Q}(\sqrt{-d})$ with class number 3 we again find that the sum $\zeta_{0}(2)+2 \zeta_{1}(2)=$ $\zeta_{K}(2)$ has a complicated expression as a combination of dilogarithms of numbers in $K$, but that the difference $\zeta_{0}(2)-\zeta_{1}(2)=\zeta_{F}(2) / \zeta(2)$ equals $4 \pi^{2} d^{-3 / 2} D\left(\sigma_{1}\left(\xi_{F}\right)\right)$ for some very simple element $\xi_{F}$ of the Bloch group of the totally real subfield $F$ of the Hilbert class field of $K$, e.g.:

\begin{tabular}{r|r|c}
$d$ & $F=\mathbb{Q}(\theta)$ & $\xi_{F}$ \\
\hline 23 & $\theta^{3}-\theta-1=0$ & {$[\theta]$} \\
31 & $\theta^{3}-\theta^{2}-1=0$ & {$[\theta]$} \\
59 & $\theta^{3}-2 \theta^{2}-1=0$ & $4[\theta]-2\left[-\theta^{2}\right]$ \\
83 & $\theta^{3}-2 \theta^{2}-2 \theta-1=0$ & $6[\theta]-2\left[\theta^{2}\right]$ \\
107 & $\theta^{3}-4 \theta^{2}+2 \theta-1=0$ & $8[\theta]-\left[\theta^{2}\right]$
\end{tabular}

In each of these cases, the conjecture is verified for $m=2$ with $\xi_{2, K}=\frac{1}{3} \xi_{K}+\frac{1}{3}\left(\sigma_{1}\left(\xi_{F}\right)-\sigma_{2}\left(\xi_{F}\right)\right)$, where $\xi_{K} \in \mathcal{B}_{2}(K)$ is an element with $D\left(\xi_{K}\right)=4 \pi^{2} d^{-3 / 2} \zeta_{K}(2)$. There are also examples like $\xi_{H,-23}$ above, e.g. the combination $\xi_{H,-31}^{(j)}=19\left[-\theta_{j-1}^{2} \theta_{j}\right]-3\left[-\theta_{j-1}^{5} \theta_{j}^{3}\right]+8\left[\theta_{j-1}^{3}\right]-8\left[\theta_{j}^{3}\right]$ for $d=31$ with

$$
D\left(\xi_{H,-31}^{(j)}\right)=-\frac{3 \cdot 31^{3 / 2}}{2 \pi^{2}} \zeta_{j}(2) \quad(j=0,1,2), \quad \theta_{j}^{3}-\theta_{j}^{2}-1=0 .
$$

3. We can proceed similarly for higher polylogarithms. For instance, for $d=23$ and the trilogarithm we have

$$
\zeta_{0}(3)+2 \zeta_{1}(3)=\zeta_{K}(3)=L\left(3,\left(\frac{\dot{2}}{23}\right)\right) \zeta(3)=\frac{96 \pi^{3}}{23^{5 / 2}} \mathcal{L}_{3}(1)
$$

(which is much simpler than the corresponding formula (2) for $m=2$, the reason being that for odd $m$ the group $\mathcal{B}_{m}(K)$ coincides up to torsion with $\left.\mathcal{B}_{m}(\mathbb{Q})\right)$ and

$$
\zeta_{0}(3)-\zeta_{1}(3)=\zeta_{F}(3) / \zeta(3)=\frac{72 \pi^{3}}{23^{5 / 2}}\left(\mathcal{L}_{3}\left(\sigma_{0}\left(\beta_{2}\right)\right)-\mathcal{L}_{3}\left(\sigma_{1}\left(\beta_{2}\right)\right)\right)
$$

by Example 3 of $\S 3$, and taking linear combinations, we obtain each partial zeta function $\zeta_{j}(3)$ as a combination of trilogarithms. A different element which exhibits the Galois equivariance property stated in the conjecture above is $\xi=[\theta]-\left[\theta^{2}\right]+\left[\theta^{5}\right]$, for which we have

$$
\frac{96 \pi^{3}}{23^{5 / 2}} \mathcal{L}_{3}\left(\sigma_{j}(\xi)\right)=\zeta_{j}(3) \quad(j \in \mathbb{Z} / 3 \mathbb{Z})
$$

which is again much simpler than the corresponding formula for the dilogarithm.

4. We now consider examples with class number $h>3$, for which the partial zeta functions $\zeta_{\mathcal{A}}(s)$ can not in general be expressed in terms of Dedekind zeta functions of subfields of $H$. For 
simplicity we restrict to $d$ prime. Then $h$ is odd and $\zeta_{H}(s) / \zeta_{K}(s)$ is the square of the quotient $\zeta_{F}(s) / \zeta(s)$ which is a product of $\frac{h-1}{2} L$-series associated to characters on $\mathcal{C} \ell(F)$ :

$$
\zeta_{H}(s)=\zeta_{K}(s) L(s)^{2}, \quad \zeta_{F}(s)=\zeta(s) L(s), \quad L(s):=\prod_{\chi \in X} L_{K}(s, \chi),
$$

where $X$ denotes a set of representatives for the non-trivial characters of $\mathcal{C} \ell_{F}$ modulo complex conjugation. Again the expression for $\zeta_{K}(m)$, especially for $m$ even, is typically more complicated than the expressions for $L(m)$, so we concentrate on the latter.

For simplicity we assume that $\mathcal{C} \ell_{K}=\langle\mathcal{A}\rangle$ is cyclic. Then, denoting by $\chi$ a generator of the character group of $\mathcal{C} \ell_{F}$ and by $\zeta_{j}(s)$ the partial zeta function $\zeta_{\mathcal{A}^{j}}(s)$, we have $\zeta_{H}(s)=\zeta_{K}(s) L(s)^{2}$, $\zeta_{F}(s)=\zeta(s) L(s)$ with

$$
L(s):=\prod_{j=1}^{(h-1) / 2} L_{K}\left(s, \chi^{j}\right)= \pm \operatorname{det}\left(\left(\zeta_{i-j}(s)-\zeta_{i+j}(s)\right)_{i, j=1, \ldots,(h-1) / 2}\right),
$$

for instance, for $h=5$ we have (denoting by $\rho$ a primitive $5^{\text {th }}$ root of unity)

$$
L(s)=\prod_{j=1}^{2}\left(\zeta_{0}(s)+\left(\rho^{j}+\rho^{-j}\right) \zeta_{1}(s)+\left(\rho^{2 j}+\rho^{3 j}\right) \zeta_{2}(s)\right)=\left|\begin{array}{ll}
\zeta_{0}(s)-\zeta_{2}(s) & \zeta_{1}(s)-\zeta_{2}(s) \\
\zeta_{1}(s)-\zeta_{2}(s) & \zeta_{0}(s)-\zeta_{1}(s)
\end{array}\right| .
$$

A consequence of the conjecture is that there exist elements $\xi_{j}=-\xi_{-j} \in \mathcal{B}_{2}(F) \otimes \mathbb{Q}$ such that $D\left(\sigma^{i}\left(\xi_{j}\right)\right)=\frac{d^{3 / 2}}{16 \pi^{2}}\left(\zeta_{i-j}(2)-\zeta_{i+j}(2)\right)$ for all $i$ and $j$, where $F$ is considered as embedded as a subfield of $\mathbb{C}$ via the real embedding and $\sigma$ is the generator of $\operatorname{Gal}(H / K)$ corresponding to $\mathcal{A}$. (Take $\xi_{j}=\sigma^{-j}(\xi)-\sigma^{j}(\xi)$ with $\xi$ as in the conjecture; by the first remark following the conjecture, this can also be written as $(1+c) \sigma^{j}(\xi)$ with $c$ the generator of $\operatorname{Gal}(H / F)$, and hence belongs to $\mathcal{B}_{2}(F)$.) The conjecture is easier to check in this form than in the original one, because (as we already saw in the $h=3$ examples) the value of $\zeta_{K}(2)=\sum_{j} \zeta_{j}(2)$ is typically much harder to express in terms of dilogarithms than the differences $\zeta_{i}(2)-\zeta_{j}(2)$.

As a numerical example (one of many we have computed), consider the field $K=\mathbb{Q}(\sqrt{-47})$ with class number 5 . Its partial zeta functions are $\zeta_{j}(s)=\zeta_{K, \mathcal{A}^{j}}(s)=\zeta_{Q_{j}}(s), j=-2, . ., 2$, with $\mathcal{A}=\left[2, \frac{-1+\sqrt{-47}}{2}\right], \mathcal{A}^{2}=\left[3, \frac{1+\sqrt{-47}}{2}\right]$, and

$$
Q_{0}(x, y)=x^{2}+x y+12 y^{2}, \quad Q_{ \pm 1}(x, y)=2 x^{2} \pm x y+6 y^{2}, \quad Q_{ \pm 2}(x, y)=3 x^{2} \mp x y+4 y^{2} .
$$

The Hilbert class field of $K$ is $H=K(\alpha)$, where $\alpha^{5}+2 \alpha^{4}+2 \alpha^{3}+\alpha^{2}-1=0$. Taking for $\alpha$ the real root $0.5764 \ldots$ gives the real embedding of the subfield $F=\mathbb{Q}(\alpha)$. The other embeddings of $F$ into $\mathbb{C}$ send $\alpha$ to $\sigma(\alpha)=-1.105 \ldots+0.5954 \ldots i, \sigma^{2}(\alpha)=-0.1828 \ldots+1.032 \ldots i, \sigma^{3}(\alpha)=\overline{\sigma^{2}(\alpha)}$ and $\sigma^{4}(\alpha)=\overline{\sigma(\alpha)}$, where $\sigma$ is a generator of $\operatorname{Gal}(H / K)$. The group of units of $F$ is generated (up to torsion) by $\alpha$ and $\beta:=\alpha+1$, and the dilogarithms of the conjugates of the elements

$$
\xi_{1}=-\left[\alpha^{-1} \beta\right]-\left[\alpha^{3} \beta^{2}\right], \quad \xi_{2}=\left[\alpha^{4} \beta\right]+\left[\alpha^{3} \beta^{3}\right]
$$

in $\mathcal{B}_{2}(F)$ are related to the values at $s=2$ of the partial zeta functions by

$$
\begin{gathered}
\left(\begin{array}{cc}
D\left(\sigma\left(\xi_{1}\right)\right) & D\left(\sigma^{2}\left(\xi_{1}\right)\right) \\
D\left(\sigma\left(\xi_{2}\right)\right) & D\left(\sigma^{2}\left(\xi_{2}\right)\right)
\end{array}\right)=\left(\begin{array}{ll}
1.7702513872 \ldots & 0.2095840007 \ldots \\
0.2095840007 \ldots & 1.5606673865 \ldots
\end{array}\right) \\
=\frac{47^{5 / 2}}{16 \pi^{2}}\left(\begin{array}{ll}
\zeta_{0}(2)-\zeta_{2}(2) & \zeta_{1}(2)-\zeta_{2}(2) \\
\zeta_{1}(2)-\zeta_{2}(2) & \zeta_{0}(2)-\zeta_{1}(2)
\end{array}\right) .
\end{gathered}
$$




\section{Known results for $m=2$}

For $m=2$ the conjecture described in $\S 5$ can be formulated in terms of $K$-theory, since we know that the Bloch group $\mathcal{B}_{2}(H)$ is isomorphic to $K_{3}(H)$ and that the dilogarithm regulator map coincides with the Borel regulator. An abstract proof in this case has been given by Deninger [15], [16], who showed the existence of an element of $K_{3}$ whose regulator is given by the special values at $s=2$ of the partial zeta functions of $K$.

The problem of constructing explicit elements $\xi \in \mathcal{B}_{2}(\overline{\mathbb{Q}})$ (though not necessarily in $\mathcal{B}(H)$ ) with the required image under $D$ was solved very recently by A. Levin. To each ideal $\mathfrak{a}$ in the ideal class $\mathcal{A}$ of $K$ there is associated an elliptic curve $E=\mathbb{C} / \mathfrak{a}$ defined over $H$ with complex multiplication by $\mathcal{O}_{K}$. For any three points $a, b, c \in E$, Levin considers the quantity

$$
\gamma_{a, b, c}=\frac{\wp_{a}-\wp_{c}}{\wp_{a}-\wp_{b}}
$$

Here $\wp_{a}=\wp_{E}(a)$ is the value of the Weierstrass $\wp$-function of $E$ at $a$. If $a, b$ and $c$ are torsion points, then $\gamma_{a, b, c}$ belongs to a certain abelian extension $H^{\prime} \supseteq H$ of $K$ (a ray class field).

Proposition (Levin). Let $\mathfrak{a}$ be an ideal class in the imaginary quadratic field $K$ and $\lambda \in \mathcal{O}_{K}$, $\mu=1-\lambda$. Then the element

$$
\xi_{\mathcal{A}, \lambda}=4 N(\lambda) N(\mu)[\lambda]+\sum_{\substack{\alpha \in \lambda^{-1} \mathfrak{a} / \mathfrak{a}-\{0\} \\ \beta \in \mu^{-1} \mathfrak{a} / \mathfrak{a}-\{0\}}} \sum_{\substack{l(\bmod N(\lambda)) \\ m(\bmod N(\mu))}}\left[\gamma_{\alpha, \beta, l \alpha+m \beta}\right]
$$

of $\mathbb{Z}\left[H^{\prime}\right]$ belongs to $\mathcal{B}\left(H^{\prime}\right)$.

The key point in proving this is that $\gamma_{a, b, c}$ and $1-\gamma_{a, b, c}$ have a factorization

$$
\gamma_{a, b, c}=\frac{\sigma_{a-c} \sigma_{a+c} \sigma_{b}^{2}}{\sigma_{a-b} \sigma_{a+b} \sigma_{c}^{2}}, \quad 1-\gamma_{a, b, c}=\frac{\sigma_{b-c} \sigma_{b+c} \sigma_{a}^{2}}{\sigma_{b-a} \sigma_{b+a} \sigma_{c}^{2}}
$$

in terms of the Weierstrass $\sigma$-function. Using this, one can write the image of each term in the sum under $\beta_{2}$ and show that the sum maps to 0 . Moreover, from the theory of complex multiplication one sees fairly easily that $\xi_{\mathcal{A}, \lambda}$ depends only on the ideal class $\mathcal{A}$ of $\mathfrak{a}$ (as the notation suggests), is invariant under $\operatorname{Gal}\left(H^{\prime} / H\right)$, and transforms under $\operatorname{Gal}(H / K)$ by $\sigma_{\mathcal{A}}\left(\xi_{\mathcal{B}, \lambda}\right)=\xi_{\mathcal{A B}, \lambda}$. The general polylogarithm conjecture now suggests that the image of $\xi_{\mathcal{A}, \lambda}$ under $D$ is a multiple of $\zeta_{K, \mathcal{A}}(2)$. Numerical experiments suggested what the correct multiple should be, and the resulting conjectural formula was then proved in [33].

Theorem (Levin). With the above notation,

$$
D\left(\xi_{\mathcal{A}, \lambda}\right)=(N(\lambda)+1)(N(\mu)+1) \cdot \zeta_{K, \mathcal{A}}(2) .
$$

The proof depends on relating the value of the dilogarithms to the values of a certain "elliptic (1,1)-logarithm" related to the elliptic polylogarithms which will be discussed in Part III. Nothing in the construction of the element $\xi_{\lambda}$ or the proof of the theorem depends in a really essential way on the fact that $m=2$, and it can be hoped that Levin's construction will be generalized to give explicit elements of $\mathcal{B}_{m}\left(H^{\prime}\right)^{\mathrm{Gal}\left(H^{\prime} / H\right)} \otimes \mathbb{Q}$ whose values under the $m^{\text {th }}$ polylogarithm regulator map are as given in (15). 


\section{Enhanced partial zeta values and a new invariant for imaginary quadratic fields}

A particular amusing consequence of the conjecture becomes apparent if we apply the "lifting" of $\mathcal{L}_{m}$ as described in $\S 3$. Again, let $K$ be imaginary quadratic with Hilbert class field $H$. By the conjecture in $\S 5$ (or, for $m=2$, the theorems of Deninger and Levin just quoted), we have for each $m$ a canonical element $\xi_{m, K} \in \mathcal{B}_{m}(H)$ satisfying (15). But from the discussion in $\S 4$ we know that on the $m^{\text {th }}$ Bloch group there is a natural lifting of the function $\mathcal{L}_{m}$ to an enhanced function $\widehat{\mathcal{L}}_{m}$ with values in $\mathbb{C} / \mathbb{Q}(m)$. It is then natural to ask the

Questions: 1. Is there a naturally defined invariant $I_{m}(\mathcal{A})$ belonging to $\mathbb{C} / \mathbb{Q}(m)$ (or even $\mathbb{C} / \alpha_{m} \mathbb{Z}(m)$ for some $\alpha_{m} \in \mathbb{Q}$ independent of $\left.K\right)$ such that

$$
\Re_{m}\left(I_{m}(\mathcal{A})\right)= \pm \frac{\left|D_{K}\right|^{m-\frac{1}{2}}(m-1) !}{2(2 \pi)^{m}} \zeta_{K, \mathcal{A}}(m) \quad ?
$$

2. If so, does the "enhanced higher Kronecker limit formula" $\widehat{\mathcal{L}}_{m}\left(\sigma_{\mathcal{A}}\left(\xi_{m, K}\right)\right)=I_{m}(\mathcal{A})$ hold?

In this paragraph we show that the answers to 1. and 2. are "Yes" and "Experimentally, yes."

Construction of the invariant $I_{m}(\mathcal{A})$. We had already observed that $\zeta_{K, \mathcal{A}}(s)$ for imaginary quadratic $K$ and an ideal class $\mathcal{A}$ in $K$ is expressible in terms of the non-holomorphic Eisenstein series $E(z, s)$ of weight 0 by (16), where the argument $z$ is the root in the upper halfplane of $a z^{2}+b z+c=0$ for some positive definite binary quadratic form $Q(p, q)=a p^{2}+b p q+c q^{2}$ corresponding to an ideal in the ideal class $\mathcal{A}$. The above question can therefore be rephrased:

Can the value of $\pi^{-m}|D|^{(m-1) / 2} E(z, m) \in \mathbb{R}(m=2,3,4, \ldots)$ be lifted in a natural way to $\mathbb{C} / \mathbb{Q}(m)$ when $z \in \mathbb{H} / \Gamma$ is a CM-point of discriminant $D$ ?

To do this, we look at the holomorphic Eisenstein series

$$
G_{2 m}(z)=\frac{1}{2} \zeta(1-2 m)+\sum_{n=1}^{\infty} \sigma_{2 m-1}(n) e^{2 \pi i n z}
$$

of weight $2 m$ on $\mathrm{SL}_{2}(\mathbb{Z})$. (Here $\sigma_{\nu}(n):=\sum_{d \mid n} d^{\nu}$.) Its Eichler integral is any function

$$
\widetilde{G}_{2 m}(z)=\frac{1}{2} \zeta(1-2 m) \cdot \frac{(2 \pi i z)^{2 m-1}}{(2 m-1) !}+\sum_{j=0}^{2 m-2} a_{j} z^{j}+\sum_{n=1}^{\infty} \sigma_{1-2 m}(n) e^{2 \pi i n z}
$$

$\left(a_{0}, \ldots, a_{2 m-2}\right.$ for the moment arbitrary). From $\left(\frac{1}{2 \pi i} \frac{d}{d z}\right)^{2 m-1} \widetilde{G}_{2 m}=G_{2 m}$ and the modularity of $G_{2 m}$ it follows, as is well-known, that

$$
(c z+d)^{2 m-2} \widetilde{G}_{2 m}\left(\frac{a z+b}{c z+d}\right)=\widetilde{G}_{2 m}(z)+P_{m, \gamma}(z) \quad \forall \gamma=\left(\begin{array}{ll}
a & b \\
c & d
\end{array}\right) \in \Gamma
$$

for some polynomial $P_{m, \gamma}$ of degree $\leq 2 m-2$. The important point is that if we choose

$$
a_{0}=\frac{1}{2} \zeta(2 m-1), \quad a_{1}=\cdots=a_{n-2}=0
$$

(this is not the only possible choice; see below), then the coefficients of the polynomials $P_{m, \gamma}(z)$ lie in $\mathbb{Q}(2 m-1)$ for all $\gamma \in \Gamma$.

We now apply to $\widetilde{G}_{2 m}$ the differential operator

$$
\mathbb{D}_{m}=\partial_{-2} \circ \partial_{-4} \circ \cdots \circ \partial_{2-2 m}=\sum_{\ell=0}^{m-1} \frac{(m+\ell-1) !}{\ell !(m-\ell-1) !}\left(\frac{1}{4 \pi y}\right)^{\ell}\left(\frac{1}{2 \pi i} \frac{d}{d z}\right)^{m-1-\ell}
$$


where $\partial_{k}=\frac{1}{2 \pi i} \frac{\partial}{\partial z}-\frac{k}{4 \pi y}$, which has the property of mapping modular forms of weight $k$ to modular forms of weight $k+2$. The result is a new function

$$
\mathcal{E}_{m}(z)=\mathbb{D}_{m}\left(\widetilde{G}_{2 m}(z)\right)
$$

which satisfies

$$
\mathcal{E}_{m}(\gamma z)=\mathcal{E}_{m}(z)+P_{m, \gamma}^{*}\left(\frac{1}{2 i y}, \frac{x}{i y}, \frac{|z|^{2}}{2 i y}\right) \quad \text { for all } \gamma \in \Gamma
$$

where $P_{m, \gamma}^{*}$ is a homogeneous polynomial of degree $m-1$ with coefficients in $\mathbb{Q}(m)$. This has two consequences:

(i) The real part of $\mathcal{E}_{m}(z)$ is $\Gamma$-invariant. Using the explicit Fourier expansions, one shows that

$$
\Re\left(\mathcal{E}_{m}(z)\right)=\frac{(m-1) !}{2 \pi^{m}} E(z, m) \quad(z \in \mathbb{H}) .
$$

(ii) If $z$ satisfies a quadratic equation $a x^{2}+b x+c=0$ with $a, b, c \in \mathbb{Z}$ and discriminant $D=b^{2}-4 a c<0$, then the image of $D^{(m-1) / 2} \mathcal{E}_{m}(z)$ in $\mathbb{C} / \mathbb{Q}(m)$ is unchanged under $z \mapsto \gamma z$, $\gamma \in \Gamma$, because $D^{(m-1) / 2} P_{m, \gamma}^{*}\left(\frac{1}{2 i y}, \frac{x}{i y}, \frac{|z|^{2}}{2 i y}\right)=P_{m, \gamma}^{*}(a, b, c) \in \mathbb{Q}(m)$.

It follows from (i) and (ii) that the invariant

$$
I_{m}(\mathcal{A})=D^{(m-1) / 2} \mathcal{E}_{m}\left(z_{\mathcal{A}}\right)
$$

where $z_{\mathcal{A}}$ is the image in $\mathbb{H} / \Gamma$ of $z_{Q}$ for any quadratic form $Q$ in the class of $\mathcal{A}$, is well defined modulo $\mathbb{Q}(m)$ (and even modulo $\alpha_{m} \mathbb{Z}(m)$ for some $\alpha_{m} \in \mathbb{Q}$ independent of $D$ ) and satisfies (19). Observe that by changing the choices of $a_{j}$ in $(20)$ by elements of $\mathbb{Q}(2 m-1)$ we change the cocycle $\gamma \mapsto P_{m, \gamma}$ by a coboundary with coefficients in $\mathbb{Q}(m-1)$; this does not affect the property that the indeterminacy of $I_{m}(\mathcal{A})$ belongs to $\mathbb{Q}(m)$, but can change the specific lattice $\mathbb{Z}(m) \alpha_{m}$.

Explicit formulas for $I_{m}(\mathcal{A})$. For $m=2$ we have the Fourier expansions

$$
\begin{gathered}
E(z, 2)=\frac{\pi^{4} y^{2}}{90}+\frac{\pi \zeta(3)}{2 y}+\frac{\pi}{y} \sum_{n=1}^{\infty} \sigma_{-3}(n)(1+2 \pi n y) e^{-2 \pi n y} \cos (2 \pi n x) \\
G_{4}(z)=\frac{1}{240}+\sum_{n=1}^{\infty} \sigma_{3}(n) e^{2 \pi i n z}, \quad \widetilde{G}_{4}(z)=\frac{(2 \pi i z)^{3}}{1440}+\frac{\zeta(3)}{2}+\sum_{n=1}^{\infty} \sigma_{-3}(n) e^{2 \pi i n z} \\
\mathcal{E}_{2}(z)=\frac{(2 \pi i z)^{2}(i z+3 y)}{1440 y}+\frac{\zeta(3)}{4 \pi y}+\sum_{n=1}^{\infty} \sigma_{-3}(n)\left(n+\frac{1}{2 \pi y}\right) e^{2 \pi i n z} .
\end{gathered}
$$

The function $\mathcal{E}_{2}(z)$ has real part $\frac{1}{2 \pi^{2}} E(z, 2)$ and satisfies the modular transformation formulas

$$
\mathcal{E}_{2}(z+1)=\mathcal{E}_{2}(z)+\pi^{2} \frac{|z|^{2}+x+1 / 3}{120 i y}, \quad \mathcal{E}_{2}(-1 / z)=\mathcal{E}_{2}(z)-\frac{\pi^{2} x}{72 i y}
$$

(the proof of (26) will be indicated below). It follows that the invariant $I_{2}(Q):=\sqrt{D} \mathcal{E}_{2}\left(z_{Q}\right)$ satisfies (19) and

$$
I_{2}([a, b+2 a, c+b+a])=I_{2}([a, b, c])+\frac{\pi^{2}}{60}\left(c-\frac{b}{2}+\frac{a}{3}\right), \quad I_{2}([c,-b, a])=I_{2}([a, b, c])-\frac{\pi^{2}}{72} b
$$


and hence that

$$
I_{2}(\mathcal{A})=I_{2}([Q]) \in \mathbb{C} / \frac{\pi^{2}}{360} \mathbb{Z} \quad \text { is well-defined } .
$$

We can improve upon the number 360 in the denominator by choosing a slightly different normalization:

$$
\mathcal{E}_{2}^{\bullet}(z)=\mathcal{E}_{2}(z)+\frac{i \pi^{2}}{240} \frac{|z|^{2}+5 x / 3+1}{y}
$$

(corresponding to replacing $\widetilde{G}_{4}$ by $\widetilde{G}_{4}+\frac{i \pi^{3}}{120}\left(z^{2}+\frac{5}{3} z+1\right)$ ), which still has real part $\left(2 \pi^{2}\right)^{-1} E(z, 2)$ but now transforms under $S L_{2}(\mathbb{Z})$ by

$$
\mathcal{E}_{2}^{\bullet}(z+1)=\mathcal{E}_{2}^{\bullet}(z)+\frac{i \pi^{2}}{120} \frac{|z|^{2}-1}{y}, \quad \mathcal{E}_{2}^{\bullet}(-1 / z)=\mathcal{E}_{2}^{\bullet}(z),
$$

so that the invariant

$$
I_{2}^{\bullet}(Q):=\sqrt{D} \mathcal{E}_{2}^{\bullet}\left(\frac{b+\sqrt{D}}{2 a}\right)=I_{2}(Q)-\frac{\pi^{2}}{24}\left(\frac{a+c}{5}-\frac{b}{6}\right)
$$

satisfies

$$
I_{2}^{\bullet}([a, b+2 a, c+b+a])=I_{2}^{\bullet}([a, b, c])+\frac{\pi^{2}(a-c)}{60}, \quad I_{2}^{\bullet}([c,-b, a])=I_{2}^{\bullet}([a, b, c])
$$

and hence gives a class invariant $I_{2}^{\bullet}(\mathcal{A})=I_{2}^{\bullet}([Q])$ which is well-defined modulo $\frac{\pi^{2}}{60} \mathbb{Z}$.

For $m>2$ the formulas are more complicated. We give only the Fourier expansion of the function $\mathcal{E}_{m}(z)$ :

$$
\mathcal{E}_{m}(z)=\sum_{\ell=0}^{m-1} \frac{(m+\ell-1) !}{\ell !(m-\ell-1) !}(4 \pi y)^{-\ell}\left[\frac{\zeta(1-2 m)}{2} \frac{(2 \pi i z)^{\ell+m}}{(\ell+m) !}+\sum_{n=0}^{\infty} n^{m-1-\ell} \sigma_{1-2 m}(n) q^{n}\right],
$$

where $\sigma_{s}(0):=\frac{1}{2} \zeta(-s)$. The modular transformation properties of this can be given explicitly, but for $m>2$ we do not know how to add a coboundary to get a function $\mathcal{E}_{m}^{\bullet}(z)$ with an optimal denominator in its transformation law with respect to 27.

We explain briefly how to prove the transformation formulas $(26)$. (The calculation for $\mathcal{E}_{m}$ goes the same way.) These formulas follow by applying $\mathbb{D}_{2}=\partial_{-2}$ to the transformation properties

$$
\widetilde{G}_{4}(z+1)=\widetilde{G}_{4}(z)-\frac{\pi^{3} i\left(3 z^{2}+3 z+1\right)}{180}, \quad z^{2} \widetilde{G}_{4}(-1 / z)=\widetilde{G}_{4}(z)+\frac{\pi^{3} i z}{36} .
$$

The first of these equations is evident since $\widetilde{G}_{4}$ is the sum of a periodic function and a multiple of $z^{3}$. For the second, we observe that the difference $z^{2} \widetilde{G}_{4}(-1 / z)-\widetilde{G}_{4}(z)$ is equal to a polynomial of degree $\leq 2, A z^{2}+B z+C$. Interchanging the role of $z$ and $-1 / z$, one deduces immediately $C=-A$. To compute the values, the easiest way is to notice that the function

$$
g(y)=\sum_{n=1}^{\infty} \sigma_{-3}(n) e^{-2 \pi n y}=\widetilde{G}_{4}(i y)-\frac{\zeta(3)}{2}+\frac{\pi^{3}}{180} y^{3}
$$

satisfies the transformation law

$$
g(y)=-y^{2} g(1 / y)+A_{-1} y^{-1}+A_{0}+A_{1} y+A_{2} y^{2}+A_{3} y^{3}
$$

with $A_{-1}=A_{3}=\frac{\pi^{3}}{180}, A_{0}=A_{2}=A-\frac{1}{2} \zeta(3), A_{1}=-i B$. The fact that $g$ is exponentially small at $\infty$ then implies that the Mellin transform $\widetilde{g}(s)=\int_{0}^{\infty} g(y) y^{s-1} d y$ converges for all $\Re(s) \gg 0$ and has a meromorphic continuation with simple poles of residue $A_{\nu}$ at $s=-\nu(\nu=-1, \ldots, 3)$ and no other poles. But $\widetilde{g}(s)$ is easily computed to be $(2 \pi)^{-s} \Gamma(s) \zeta(s) \zeta(s+3)$, and by comparing the residues we find $A=C=0, B=i \pi^{3} / 36$. 
The enhanced conjecture. Now that we have defined the invariant $I_{m}(\mathcal{A})$, we can formally state the conjecture which was suggested at the beginning of the section.

Conjecture. Let $K$ be an imaginary quadratic field with Hilbert class field $H, m \geq 2$. Then the numbers $I_{m}(\mathcal{A}) \in \mathbb{C} / \mathbb{Q}(m)$ are equal to the enhanced polylogarithms $\widehat{\mathcal{L}}_{m}\left(\sigma_{\mathcal{A}}(\xi)\right)$ for some element $\xi \in \mathcal{B}_{m}(H) \otimes \mathbb{Q}$.

More precisely, we should have $N_{m} \xi \in \mathcal{B}_{m}(H)$ and $\widehat{\mathcal{L}}_{m}(\xi) \equiv I_{m}(\mathcal{A}) \bmod N_{m}^{-1} \mathbb{Z}(m)$ for some positive integer $N_{m}$ depending on $m$ but not on $K$.

Examples. 1. We consider our usual example $K=\mathbb{Q}(\sqrt{-23})$ and, for $m=2$, the element $\xi=\xi_{2, K} \in \mathcal{B}_{2}(K)$ constructed in Example 1 of $\S 5$. The real part of $I_{2}\left(\mathcal{A}_{0}\right)$ is a rational multiple of $\pi^{2}$ and hence not interesting (this follows either by substituting $z_{0}=\frac{1+\sqrt{-23}}{2}$ into the Fourier expansion of $\mathcal{E}_{2}(z)$ given above or else by noting that $I_{2}\left(\mathcal{A}^{-1}\right)=-\overline{I_{2}(\mathcal{A})}$ modulo $\mathbb{Q}(2)$ for any ideal class $\mathcal{A}$ ). For $\mathcal{A}_{1}$ we find by $(23)$ and $(25)$

$$
I_{2}\left(\mathcal{A}_{1}\right) \equiv \alpha+\frac{23^{3 / 2}}{8 \pi^{2}} \zeta_{1}(2) \cdot i(\bmod \mathbb{Q}(2))
$$

with

$$
\begin{aligned}
\alpha & =\frac{7 \pi^{2}}{576}-\frac{2}{\pi} \sum_{n=0}^{\infty}(-1)^{n} \sigma_{-3}(2 n+1)\left(1+\pi\left(n+\frac{1}{2}\right) \sqrt{23}\right) e^{-\pi\left(n+\frac{1}{2}\right) \sqrt{23}} \\
& =0.11703683929364456681742552529827002050121888426989 \ldots .
\end{aligned}
$$

The numbers $\Re\left(\widehat{D}\left(\theta_{0}\right)\right)=2.10466 \ldots$ and $\Re\left(\widehat{D}\left(\theta_{1}\right)\right)=-11.7444 \ldots$ appearing in Example 3 of $\S 4$ are equal (to high accuracy) to $\alpha+\frac{29}{144} \pi^{2}$ and $-\frac{1}{2} \alpha-\frac{341}{288} \pi^{2}$, respectively, and this verifies the above conjecture with $\xi$ as given in equation (18).

2. Similarly, for $m=3$, we find that the invariants $I_{3}\left(\mathcal{A}_{j}\right)$ are given by

$$
\begin{aligned}
& I_{3}\left(\mathcal{A}_{0}\right) \equiv-\frac{23^{2}}{4 \pi^{3}} \zeta_{0}(3)-\frac{N_{0}}{7560} \pi^{3} \cdot i(\bmod \mathbb{Q}(3)) \\
& I_{3}\left(\mathcal{A}_{1}\right) \equiv-\frac{23^{2}}{4 \pi^{3}} \zeta_{1}(3)+\left(-4 \cdot f+\frac{N_{1}}{3780} \pi^{3}\right) \cdot i(\bmod \mathbb{Q}(3))
\end{aligned}
$$

where $f=15.1353 \ldots$ is the number given in the example at the end of $\S 4, N_{0}=1021$ and $N_{1}=7319$ to high accuracy. The proportionality of $\zeta_{0}(3)-\zeta_{1}(3)$ with $\mathcal{L}_{3}\left(\beta_{1}\right)$ verified in $\S 4$ thus lifts (experimentally) to the enhanced values, as predicted. 


\section{PART III. ElliptiC CURVES AND ELLIPTIC POLYLOGARITHMS}

\section{The elliptic dilogarithm conjecture}

The elliptic dilogarithm and $L(E, 2)$. The elliptic dilogarithm $D^{E}$ associated to the elliptic curve $E / \mathbb{C}$, as introduced by Bloch [6], is an "averaged" version of the Bloch-Wigner dilogarithm $D$ : more precisely one represents $E(\mathbb{C})$ as the Tate curve $\mathbb{C}^{\times} / q^{\mathbb{Z}}$ and averages $D$ over the action of $\mathbb{Z}$ on $\mathbb{C}^{\times}$, i.e.

$$
D^{E}(P)=\sum_{l=-\infty}^{\infty} D\left(q^{l} x\right),
$$

where $P \in E(\mathbb{C})$ is the image of $x \in \mathbb{C}^{\times}$.

Since the series is invariant under $x \mapsto q x$ and converges absolutely, this is independent of the choice of $x$. The series converges rapidly, so that $D^{E}(P)$ is easily calculable. Another representation of $D^{E}(P)$ is given by writing $E=\mathbb{C} / \mathbb{Z} \tau+\mathbb{Z}, \tau \in \mathbb{H}\left(\right.$ so $q=e^{2 \pi i \tau}$ ) and $P=z$ $(\bmod \mathbb{Z} \tau+\mathbb{Z})\left(\right.$ and so $\left.x=e^{2 \pi i z}\right) ;$ then $D^{E}$ is given by the Kronecker-Eisenstein series

$$
D^{E}(P)=\frac{\Im(\tau)^{2}}{\pi} \Re\left(\sum_{m, n \in \mathbb{Z}}^{\prime} \frac{\exp (2 \pi i(n \xi-m \eta))}{(m \tau+n)^{2}(m \bar{\tau}+n)}\right)
$$

where $z=\xi \tau+\eta$, as one shows by computing the Fourier expansion of the right-hand side with respect to $\tau$ (cf. [6] or [54]).

The $L$-series of $E$ at the point $s=1$ is well-known to be linked with the height pairing on the rational points of $E$. On the other hand, this pairing is given in terms of logarithms of theta functions which can be seen as the elliptic analogue of the logarithm. It was Bloch's idea that $L(E / \mathbb{Q}, 2)$ might be linked to the elliptic dilogarithm, in the following way

$$
L(E / \mathbb{Q}, 2) \stackrel{?}{=} \pi \times \text { rational linear combination of } D^{E}\left(P_{i}\right), \quad P_{i} \in E(\overline{\mathbb{Q}}) .
$$

This was proved by him for CM elliptic curves and by Beilinson [2] for modular curves (see below). However, these authors considered only torsion points on $E(\overline{\mathbb{Q}})$. This would be the analogue in the number field case of considering values of polylogarithms only at roots of unity, which would suffice to describe $\zeta_{F}(m)$ for all abelian $F$, but would not lead to the discovery of the higher Bloch groups $\mathcal{B}_{m}(F)$ and the corresponding conjectural description of $K_{2 m-1}(F)$.

This consideration suggests that one should look for conditions describing linear combinations of points on $E$ such that the elliptic dilogarithm evaluated on them gives a rational multiple of the $L$-value. Numerical experiments carried out some 10 years ago with $H$. Cohen suggested that one had

$$
\sum_{i} n_{i} D^{E}\left(P_{i}\right) \in \mathbb{Z} \cdot R_{E}, \quad R_{E}=\frac{N}{8 \pi} L(E, 2)
$$

( $N=$ conductor of $E$ ), whenever the divisor $\sum n_{i}\left(P_{i}\right)$ satisfies the following conditions:

(i) $\sum n_{i} P_{i}=0$ on $E$

(ii) $\sum n_{i}\left(P_{i}\right)^{3}=0$ in $\operatorname{Sym}^{3}(E)$

(iii) $\sum n_{i} \lambda_{p}\left(P_{i}\right) P_{i}=0$ on $E$ for any prime $p$. 
Here $\lambda_{p}(P)$ is defined as the power of $p$ dividing $c$, where $c^{2}$ and $c^{3}$ are the denominators of $x(P)$ and $y(P)$ in a minimal model of the curve $E$. Note that $R_{E}=\frac{1}{2} \pi\left|L^{\prime}(E, 0)\right|$ if $E / \mathbb{Q}$ is modular, by the functional equation of $L(E, s)$.

Example. The modular curve $X_{0}^{*}(37)$ has the minimal model $E=E_{37}: y^{2}-y=x^{3}-x$. The Mordell-Weil group $E(\mathbb{Q})$ is infinite cyclic with generator $P=(0,0)$. We have five multiples of $P$ which are integral

$$
P=[0,0], 2 P=[1,0], 3 P=[-1,1], 4 P=[2,3], 6 P=[6,-14],
$$

(as well, of course, as their negatives) and two further multiples which involve only the single prime " 2 " in their denominators:

$$
5 P=\left[\frac{1}{4}, \frac{5}{8}\right], 10 P=\left[\frac{161}{16}, \frac{2065}{64}\right] .
$$

For each $k$, the combination

$$
\xi_{k}=(k P)-k(P)-\frac{k^{3}-k}{6}((2 P)-2(P))
$$

satisfies the conditions (i) and (ii) above. For $k=3,4$ and 6 the combinations

$$
\xi_{3}=(3 P)-4(2 P)+5(P), \quad \xi_{4}=(4 P)-10(2 P)+16(P), \quad \xi_{6}=(6 P)-35(2 P)+64(P)
$$

also satisfy (iii), since all the points involved are integral. The combinations $\xi_{5}$ and $\xi_{10}$ do not separately satisfy (iii) for $p=2$, but the combination

$$
\xi_{10}^{*}=\xi_{10}-4 \xi_{5}=(10 P)-4(5 P)-85(2 P)+180(P)
$$

does, since $\lambda_{2}(5 P)=1, \lambda_{2}(10 P)=2$. Computing numerically to high precision, we find:

$$
D^{E}\left(\xi_{3}\right)=8 R_{E}, \quad D^{E}\left(\xi_{4}\right)=26 R_{E}, \quad D^{E}\left(\xi_{6}\right)=90 R_{E}, \quad D^{E}\left(\xi_{10}^{*}\right)=248 R_{E},
$$

with $R_{E}=\frac{37}{8 \pi} L(E, 2)=0.561748914579 \ldots$

In the general case, conditions (i)-(iii) are not quite correct, as was pointed out by Schappacher-Rolshausen [40] and others. On the one hand, one needs an extra condition at certain primes of bad reduction (namely for $p$ of split multiplicative reduction such that the Néron model is an $N$-gon with $N>1$ ). On the other hand, (i) is not always necessary. For example (due to $\mathrm{K}$. Rolshausen in his thesis), if $E$ is the curve $y^{2}+y=x^{3}-325 x+6156$ of conductor 4025 , then the combination $16(P)-10(2 P)+(4 P)$ for $P=(20,87)$ satisfies (i)-(iii) above but fails to give a rational multiple of the regulator, while $11(Q)+2(2 Q)-(3 Q)$ for $Q=(45,287)$ does not satisfy (i) but nevertheless maps under $D^{E}$ to $\frac{4025}{8 \pi} L(E, 2)$.

The right way is to replace $\lambda_{p}(P)$ in (iii) by $h_{p}(P)$, the local height at $p$ in $P$. Here $h_{p}$ denotes the local height associated to the valuation $p$. In this form, it is independent of the model of $E$ used. For $P$ of good reduction, this is just $\lambda_{p}(P) \log p$, so there is no change, and for certain $p$ of bad reduction (like $p=37$ in the example above) the height condition at $p$ reduces to (i) if all the $P_{i}$ are $p$-integral, which is why (i) often occurred. The corrected conditions are then

(a) $\sum n_{i}\left(P_{i}\right)^{3}=0$ in $\operatorname{Sym}^{3}(E)$,

(b) $\sum n_{i} h_{p}\left(P_{i}\right) P_{i}=0$ on $E$ for any prime $p$,

(c) some integrality condition at primes $p$ of split multiplicative reduction. 
We then expect that $(29)$ holds for any $\xi=\sum n_{i}\left(P_{i}\right) \in \mathbb{Z}[E(\mathbb{Q})]$ satisfying (a)-(c).

More generally, we expect the same thing to happen for divisors $\xi=\sum n_{i}\left(P_{i}\right) \in \mathbb{Z}[E(\overline{\mathbb{Q}})]$ which are defined over $\mathbb{Q}$ (i.e. are $\operatorname{Gal}(\overline{\mathbb{Q}} / \mathbb{Q})$-invariant) but whose individual points $P_{i}$ are defined only over $\overline{\mathbb{Q}}$. (It is important to consider such more general divisors because there may not be enough points in $E(\mathbb{Q})$ to produce linear combinations satisfying (a)-(c). For instance, $E(\mathbb{Q})$ might be $\{0\}$.) We therefore define

$$
\mathcal{A}_{2}(E / \mathbb{Q})=\left\{\xi \in \sum n_{i}\left(P_{i}\right) \in \mathbb{Z}[E(\overline{\mathbb{Q}})]^{\mathrm{Gal}(\overline{\mathbb{Q}} / \mathbb{Q})} \mid \xi \text { satisfies }(\mathrm{a})-(\mathrm{c})\right\}
$$

where the words "prime $p$ " must be replaced by "archimedean or non-archimedean place $v$ " in (b) and by "non-archimedean place $v$ " in (c). (Condition (a) implies that the sum over all $v$ of the expression in (b) vanishes, so that we can always omit one place; this is why in formulating (b) for $\mathbb{Q}$ we could restrict attention to finite primes.) Then we expect:

Conjecture. The image of the map $D^{E}: \mathcal{A}_{2}(E / \mathbb{Q}) \rightarrow \mathbb{R}$ is a lattice commensurable with $\mathbb{Z} \cdot R_{E}$.

Functional equations and the elliptic Bloch group. As for the case of the usual dilogarithm, we want to find a subgroup $\mathcal{C}_{2}(E / \mathbb{Q})$ of $\mathcal{A}_{2}(E / \mathbb{Q})$ generated by some universal relations of $D^{E}$ and to conjecture that the "elliptic Bloch group" $\mathcal{B}_{2}(E / \mathbb{Q})=\mathcal{A}_{2}(E / \mathbb{Q}) / \mathcal{C}_{2}(E / \mathbb{Q})$ maps isomorphically onto the regulator lattice.

Two special relations are evident: from the inversion relation $D\left(x^{-1}\right)=-D(x)$ of the usual dilogarithm we obtain $D^{E}(P)=-D^{E}(-P)$, and from the duplication relation $D\left(x^{2}\right)=2 D(x)+$ $2 D(-x)$ we easily find that $D^{E}(2 P)=2 \sum_{T \in E[2]} D^{E}(P+T)$. (Combine $x,-x, \sqrt{q} x$ and $-\sqrt{q} x$ in the definition of $D^{E}$.) More interesting is Bloch's relation [6], whose proof will be recalled in $\S 9$, that $D^{E}$ also vanishes on the elements $\eta_{f}=(f) *(1-f)^{-}$for all $f \in \mathbb{Q}(E)$. Here $\xi * \eta^{-}$is defined for any two divisors $\xi=\sum n_{i}\left(P_{i}\right), \eta=\sum m_{j}\left(Q_{j}\right)$ as $\sum n_{i} m_{j}\left(P_{i}-Q_{j}\right)$. It is expected that these form a full set of relations, so that if we set

$$
\mathcal{C}_{2}(E / \mathbb{Q})=\left\langle\eta_{f}, \quad(P)+(-P), \quad(2 P)-2 \sum_{T \in E[2]}(P+T)\right\rangle,
$$

then the quotient

$$
\mathcal{B}_{2}(E / \mathbb{Q}):=\mathcal{A}_{2}(E / \mathbb{Q}) / \mathcal{C}_{2}(E / \mathbb{Q})
$$

should map isomorphically under $D^{E}$ to a lattice commensurable with $\mathbb{Z} \cdot R^{E}$.

As in the number field case, one can also write the group $\mathcal{B}_{2}(E / \mathbb{Q})$ as the kernel of the map $(P) \mapsto(P) \otimes P$ from $\mathcal{G}_{3}(E)$ to $\mathcal{G}_{2}(E) \otimes E$, where $\mathcal{G}_{3}(E)=\mathbb{Z}[E] / \mathcal{C}_{2}$ and $\mathcal{G}_{2}$ is the largest quotient of $\mathbb{Z}[E]$ on which all the local heights vanish, and this is part of a larger complex $\mathcal{G}_{3}(E) \rightarrow \mathcal{G}_{2}(E) \otimes E \rightarrow E \otimes \bigwedge^{2} E \rightarrow \bigwedge^{3} E$. For details see [26].

Example: For the curve $E_{37}: y^{2}-y=x^{3}-x$ investigated above, we find that the 4 functions

$$
f_{1}=y, f_{2}=x+y, f_{3}=\frac{1+y-2 x}{4}, f_{4}=\frac{x+2 y-1}{2 x}
$$

have $\left(f_{i}\right)$ and $\left(1-f_{i}\right)$ with support in $E(\mathbb{Q})$, the expressions $\eta_{i}=\eta_{f_{i}}$ being equal (up to the "trivial" relation $(x)+(-x)=0)$ to

$$
\begin{aligned}
\eta_{1} & =-(6 P)+(4 P)+8(3 P)-7(2 P)-8(P), \\
\eta_{2} & =(6 P)-5(4 P)+5(3 P)-5(2 P)+9(P), \\
\eta_{3}=-\eta_{4} & =(10 P)-3(6 P)-4(5 P)-(4 P)+6(3 P)+6(2 P)+2(P), \\
30 &
\end{aligned}
$$


respectively, as the reader can check easily by computing the divisors of $f_{i}$ and $1-f_{i}$ in each case. Hence each identity in (30) implies the remaining three ones. (That $\eta_{3}=-\eta_{4}$ is less surprising than it looks, since any $\eta_{f}$ would have to be a vector $\sum n_{i}(i P) \in \mathbb{Z} \eta_{1}+\mathbb{Z} \eta_{2}+\mathbb{Z} \eta_{3}$ with $\sum\left|n_{i}\right| \leq 27$ and there are very few such vectors.) In other examples, one can find many more such $f$. For instance, for the curve $y^{2}+x y+y=x^{3}-x^{2}-180 x+1047$ of conductor 350 with $E(\mathbb{Q})=\mathbb{Z} \cdot P$, $P=[-1,35]$, one finds 379 essentially different functions $f$ (where "essentially different" means up to the group generated by $f \mapsto 1 / f$ and $f \mapsto 1-f)$ for which $\eta_{f} \subset \mathbb{Z}[E(\mathbb{Q})]$; for each of these the element $\eta_{f}$ is (up to the relation $(-n P)=-(n P)$ ) a linear combination of the 19 points $(P)$, $(2 P), \ldots,(16 P),(18 P),(21 P),(22 P)$, but in fact the $\eta_{f}$ lie in an 11-dimensional sublattice of this 19-dimensional lattice and are all very short vectors in this sublattice, so that we obtain the same relations among the numbers $D^{E}(n P)$ in many different ways.

Remark. If we let $q \rightarrow 0$, the Tate curve $\mathbb{C}^{\times} / q^{\mathbb{Z}}$ degenerates to $\mathbb{G}_{m}(\mathbb{C})=\mathbb{C}^{\times}$. If $f$ is any rational function on $\mathbb{P}^{1}$ satisfying $f(0)=f(\infty)$, then the degeneration of the above condition $(f) *(1-f)^{-}=0$ still makes sense, with $(P)+(Q)$ meaning simply $(P \cdot Q)$, and the corresponding relation

$$
\sum_{a, b \in \mathbb{C}^{\times}} \operatorname{ord}_{a}(f) \operatorname{ord}_{b}(1-f)\left[\frac{a}{b}\right]=0
$$

remains true in the Bloch group $B_{2}(\mathbb{C})$. For the special function

$$
f(t)=\frac{(t-a)\left(t-a^{\prime}\right)\left(t-b b^{\prime}\right)}{(t-b)\left(t-b^{\prime}\right)\left(t-a a^{\prime}\right)}, \quad\left(a^{\prime}=1-a, b^{\prime}=1-b\right)
$$

this reduces modulo the trivial relations $[x]+\left[\frac{1}{x}\right]=0$ and $[x]+[1-x]=0$ to the five term relation, which is thus seen to be in a certain sense a degeneration of its elliptic analogue.

\section{The elliptic dilogarithm and the regulator map for $K_{2}(E)$}

In this section we explain some of the known theory behind the computations and conjecture discussed in $\S 8$.

The regulator map for $K_{2}$ of a curve. For any curve $X / \mathbb{C}$, the Bloch-Beilinson regulator pairing between $K_{2}(\mathbb{C}(X))$ and the space of holomorphic 1-forms on $X$ is defined on symbols $\{F, G\}, F, G \in \mathbb{C}(X)^{*}$, by

$$
\left\langle r_{2}^{X}(\{F, G\}), \omega\right\rangle=\frac{1}{2 \pi} \int_{X(\mathbb{C})} \log |F| d \log \bar{G} \wedge \omega
$$

To see that this is defined on $K$-theory we must check that the integral vanishes for $F=1-G$. This holds because $\log |F| d \log \overline{(1-F)}$ is the sum of a holomorphic differential and of the exact differential $d \Phi(F(z))$, where $\Phi(z)=\log |z| \log |1-z|-i D(z)$. If $X$ is defined over $\mathbb{Q}$, then the group $K_{2}(X / \mathbb{Q})$ is defined as a subgroup of $K_{2}(\mathbb{Q}(X))$ generated by expressions $\alpha=\sum\left\{f_{i}, g_{i}\right\}$ $\left(f_{i}, g_{i} \in \mathbb{Q}(X)\right)$ satisfying certain local conditions (vanishing of the tame symbols) at the zeros and poles of the $f_{i}$ and $g_{i}$, and there is a still smaller subgroup $K_{2}(X / \mathbb{Z}) \subset K_{2}(X / \mathbb{Q})$ defined by certain conditions on the symbols at primes of bad reduction of $X$. The Bloch-Beilinson conjecture in the case of a curve $X / \mathbb{Q}$ predicts in this case that $K_{2}(X / \mathbb{Z})$ has rank $g$, where $g$ is the genus of $X$, and that the determinant of the $g \times g$-matrix of its pairings with the lattice of holomorphic 1-forms on $X / \mathbb{Z}$ (Néron differentials) is rationally proportional to an explicit multiple of $L(X / \mathbb{Q}, 2)$ or of $L^{(g)}(X / \mathbb{Q}, 0)$. Here $L(X / \mathbb{Q}, s)$ is the Hasse-Weil zeta function, which conjecturally satisfies a functional equation under $s \rightarrow 2-s$ with $\Gamma$-factor $A^{s} \Gamma(s)^{g}$. 
The case of elliptic curves. In particular, if $E$ is an elliptic curve, $K_{2}(E / \mathbb{Z})$ should have rank 1 and the pairing of any element in this group with $d z$ should be a simple multiple of $L(E / \mathbb{Q}, 2)$. But in this case, writing $E(\mathbb{C})$ as $\mathbb{C} / \mathbb{Z} \tau+\mathbb{Z}$, we have Bloch's formula

$$
\left\langle r_{2}^{E}\{F, G\}, \omega\right\rangle=\frac{1}{\pi} D^{E}\left((F) *(G)^{-}\right)
$$

where the pairing $\xi * \eta^{-}$of divisors $\xi=\sum n_{i}\left(P_{i}\right), \eta=\sum m_{j}\left(Q_{j}\right)$ is defined as $\sum_{i, j} n_{i} m_{j}\left(P_{i}-Q_{j}\right)$. (To prove this, write $F$ and $G$ as products of Jacobi theta series, replace the theta series by their product expansion, and integrate them term by term. See e.g. [26], pp. 416-417.) One therefore expects $L(E / \mathbb{Q}, 2)$ to be expressible in terms of special values of $D^{E}$.

In the original formulation of Bloch and Beilinson, it was thought to be $K_{2}(E / \mathbb{Q})$ which should have rank 1 and map under $r_{2}^{E}$ to a multiple of $L(E / \mathbb{Q}, 2)$. Computer experiments of Bloch and Grayson [7] suggested that one has to consider the $K$-group of the Néron model $E_{\mathbb{Z}}$ of $E$ instead. Schappacher and Scholl [41] gave a precise description of the necessary local conditions. On the other hand, by studying the structure of the divisors $\sum\left(f_{i}\right) *\left(g_{i}\right)^{-}$with $\sum\left\{f_{i}, g_{i}\right\} \in K_{2}(E / \mathbb{Q})$, Goncharov and Levin showed

Theorem $\left[26\right.$, Theorem 1.2]. For any elliptic curve $E / \mathbb{Q}$ the image of $r_{2}^{E}$ on $K_{2}(E / \mathbb{Q})$ is - up to tensoring with $\mathbb{Q}$ - the same as the image of $D^{E}$ on $\mathbb{Q}$-rational divisors satisfying the conditions (a) and (b).

Now the extra condition by Schappacher and Scholl corresponds to the extra condition (c) above, so that $D^{E}\left(\mathcal{A}_{2}(E / \mathbb{Q})\right) \otimes \mathbb{Q}$ coincides with $r_{2}^{E}\left(K_{2}(E / \mathbb{Z})\right) \otimes \mathbb{Q}$ and hence is conjecturally of rank 1 .

The case of modular curves. Finally, we have to see why the generator of this regulator lattice is related to $L(E / \mathbb{Q}, 2)$. If $E$ has complex multiplication, this is easy, because the HasseWeil zeta function of $E$ is the Hecke $L$-series of a Grössencharacter for an imaginary quadratic field and its value at $s=2$ can be written more directly as a finite linear combination of KroneckerEisenstein series (28) with $P$ a torsion point ([6], [39]). Much less obvious is Beilinson's theorem that $L(E / \mathbb{Q}, 2)$ can be written as a combination of values of the function $D^{E}$ evaluated at torsion points whenever $E$ is modular. The idea of the proof is as follows. Let $X=\mathbb{H} / \Gamma$ be a modular curve and choose $F$ and $G$ to be modular units (i.e. modular functions $X \rightarrow \mathbb{P}^{1}$ with divisors concentrated at the cusps) and $\omega=f(\tau) d \tau$ for some Hecke eigenform $f \in S_{2}(\Gamma)$. Then the logarithmic derivatives of $F$ and $G$ are Eisenstein series of weight 2 on $X$ and the integral on the right of (32) can be computed by Rankin's method as a special value of the convolution of the $L$-series of $f$ with the $L$-series of a weight 2 Eisenstein series. For a suitable choice of the Eisenstein series, it equals $L(f \otimes \chi, 1) L(f, 2)$ where $\chi$ is a Dirichlet character. If $f$ corresponds to the modular elliptic curve $E$ (i.e., $f(\tau) d \tau$ is the pull-back of $d z$ with respect to a modular parametrization $X \rightarrow E$ ), then $L(f \otimes \chi, 1)$ is an algebraic multiple (non-zero for suitable $\chi$ ) of a period of $E$ and $L(f, 2)=L(E / \mathbb{Q}, 2)$. On the other hand, the regulator pairing between $\{F, G\}$ and $\omega=f(\tau) d \tau$ is the same as the pairing between the push-forward of $\{F, G\}$ and $d z$, so by (33) it follows that $\pi^{-1} L(E, 2)$ is expressed as a $\mathbb{Q}$-linear combination of values of $D^{E}$ (with arguments which are torsion points of $E$, since the divisors of $F$ and $G$ are torsion points in the Jacobian of $X_{0}(N)$ by the Manin-Drinfeld theorem). Summarizing, we have:

Theorem (Beilinson). If $E / \mathbb{Q}$ is a modular elliptic curve, then there exists an element $\xi$ of $\mathbb{Q}\left[E_{\text {tors }}\right]$ with $D^{E}(\xi)=R_{E}$.

Connection with Mahler measure. We end this section by describing a beautiful relationship, brought to light by the work of Deninger [14], Boyd [10] and Villegas [48], between the regulator map $r_{2}^{E}$ and the "Mahler measure" of certain polynomials. 
If $P\left(x_{1}, \ldots, x_{n}\right)$ is a polynomial in $n$ variables, then the (logarithmic) Mahler measure of $P$ is defined by

$$
m(P)=\int_{0}^{1} \cdots \int_{0}^{1} \log \left|P\left(e^{2 \pi i \theta_{1}}, \ldots, e^{2 \pi i \theta_{n}}\right)\right| d \theta_{1} \cdots d \theta_{n} .
$$

For $n=1$ and $P(x)=\left(x-\alpha_{1}\right) \cdots\left(x-\alpha_{n}\right), m(P)$ is equal by Jensen's formula to the measure $\sum_{j} \log \left(\max \left(\left|\alpha_{i}\right|, 1\right)\right)$ introduced by Lehmer [31] in 1928, which for irreducible $P(x) \in \mathbb{Z}[x]$ is simply the logarithm of the height of the algebraic integer $\alpha_{1}$. The definition with an integral is due to Mahler [34].

The original purpose of the Mahler measure was for results in transcendence theory and one was only interested in the approximate size of $m(P)$, but a number of calculations suggested that the actual value of $m(P)$ might in many cases be an interesting number, and in particular was sometimes connected with special values of $L$-functions. In particular, C. Smyth [42] discovered that the value of $m(P)$ for $P(x, y)=x+y+1$ and $P(x, y, z)=x+y+z+1$ are simple multiples of the Dirichlet $L$-series value $L\left(2, \chi_{3}\right)$ and the Riemann zeta-value $\zeta(3)$, respectively; and T. Chinburg [12] observed that even in certain $n=1$ examples the value of $m(P)$ is related to the value of an Artin $L$-function at $s=1$.

In 1990, Deninger [14] discovered that the Mahler measures of arbitrary polynomials with algebraic coefficients are periods of mixed motives, and more specifically, that Mahler measures of certain polynomials in two variables could be expressed in terms of the regulator map for elliptic curves. This phenomenon was then studied experimentally in great detail by Boyd [10], who discovered a number of families of examples of this type.

For $k \in \mathbb{Z}, k>0, k \neq 4$, let $P_{k}(x, y)$ be the polynomial

$$
P_{k}(x, y)=(x+y)(x y+1)-k x y \text {. }
$$

Let $E_{k}$ be the elliptic curve over $\mathbb{Q}$ defined by $P_{k}(x, y)=0$. Then one finds numerically to high precision

$$
\left|L^{\prime}\left(E_{k}, 0\right)\right|=B_{k} m\left(P_{k}\right),
$$

with $B_{k} \in \mathbb{Q}$. (Recall that $\left|L^{\prime}(E, 0)\right|=2 \pi^{-1} R_{E}=(2 \pi)^{-2} N_{E} L(E, 2)$. We can compute $L^{\prime}\left(E_{k}, 0\right)$ using standard number theory software packages and $m\left(P_{k}\right)=\int_{0}^{1} \operatorname{arccosh}\left(\frac{1}{2} k-\cos \pi x\right) d x$ by numerical integration.) A small table of the experimentally obtained values of $B_{k}$ is as follows:

\begin{tabular}{c|ccccccccccccccccccccccc}
$k$ & 1 & 2 & 3 & 5 & 6 & 7 & 8 & 9 & 10 & 11 & 12 & 13 & 14 & 15 & 16 & 17 & 18 & 19 & 20 & $\cdots$ & 198 & 199 & 200 \\
\hline$B_{k}$ & 1 & 1 & $\frac{1}{2}$ & $\frac{1}{6}$ & 2 & 2 & $\frac{1}{4}$ & 2 & 8 & 8 & $\frac{1}{2}$ & 4 & 8 & 24 & $\frac{1}{11}$ & 24 & 16 & 40 & 2 & $\cdots$ & 12416 & 23296 & 64
\end{tabular}

The explanation of Boyd's examples was worked out by Villegas [48]. (All three papersBoyd's, Deninger's, and Villegas's - are extremely interesting and are highly recommended to the reader.) In a few words, it is as follows. The symbol $\{x, y\}$ of the two generators $x$ and $y$ of $\mathbb{Q}(E)$ satisfies the necessary conditions on the tame symbol and at primes of bad reduction to define an element of $K_{2}\left(E_{\mathbb{Z}}\right)$, and then a calculation based on Jensen's formula and integration by parts reveals that the regulator $\left\langle r_{2}^{E}(\{f, g\}), \omega\right\rangle$, where $\omega$ as usual is the Néron differential of $E_{\mathbb{Z}}$, is equal to the Mahler measure $m\left(P_{k}\right)$. (The details are given in [48].) The conjecture described above then implies that $m\left(P_{k}\right)$ is a rational multiple of $\pi^{-1} L\left(E_{k} / \mathbb{Q}, 2\right)$. We remark that in this particular example the support of $(x) *(y)^{-}$, and hence the set of arguments at which $D^{E}$ is evaluated to obtain the regulator, is contained in $E_{\text {tors }}$, but that in other families introduced in [10] and analyzed in [48], such as $P_{k}(x, y)=y^{2}-x^{3}-k x y-1$, this is not the case.

In the last section of [48], Villegas shows how to interpret $m\left(P_{k}\right)$ in modular terms: if we think of $k=k(\tau)$ (where the elliptic curve defined by $P_{k}=0$ is isomorphic to $\mathbb{C} /(\mathbb{Z} \tau+\mathbb{Z})$ ) as a 
modular function, then $m\left(P_{k}\right)$ is the real part of a holomorphic function in $\mathbb{H}$ whose derivative is a modular form of weight 3 .

\section{The elliptic trilogarithm and $L\left(\operatorname{Sym}^{2}(E), 3\right)$}

Mestre and Schappacher [35] (for torsion points), Goncharov [25] and Wildeshaus [49] (in the general case) formulated conjectures expressing certain special values of the $L$-series of symmetric powers of an elliptic curve over $\mathbb{Q}$ in terms of higher elliptic polylogarithms. In this section we describe the conjecture briefly for the next case (i.e., the elliptic trilogarithm and $\operatorname{Sym}^{2}(E)$ ) and provide a numerical example. A detailed exposition is given in [25].

The trilogarithmic picture. In this case, the general Beilinson conjecture says that $L\left(\operatorname{Sym}^{2}(E), 3\right)$ should be related to a 2-dimensional lattice, since the functional equation of $L\left(\operatorname{Sym}^{2}(E), s\right)$ shows that it has a double zero at $s=0$. Similarly, the regulator is given by two functions on divisors of $E$ : the analogue of $D^{E}$, defined by

$$
\mathcal{L}_{3,1}^{E}(x)=\sum_{n \in \mathbb{Z}} \mathcal{L}_{3}\left(q^{n} x\right)
$$

and a second one (the analogue of Bloch's $J_{q}(x)$ averaging the function $J(x)=\log |x| \log |1-x|$ )

$$
\mathcal{L}_{3,2}^{E}(x)=\sum_{n=0}^{\infty} J_{3}\left(q^{n} x\right)+\sum_{n=1}^{\infty} J_{3}\left(q^{n} x^{-1}\right)+\frac{\log ^{2}|x| \log ^{2}|q / x|}{4 \log |q|}
$$

where $J_{3}(x)=\log ^{2}|x| \log |1-x|$. This function satisfies $\mathcal{L}_{3,2}^{E}(x)=\mathcal{L}_{3,2}^{E}(q x)$ because

$$
J_{3}(x)-J_{3}\left(x^{-1}\right)=-\log ^{3}|x|=\frac{\log ^{2}|q x| \log ^{2}|x|-\log ^{2}|x| \log ^{2}|q / x|}{4 \log |q|} .
$$

The functions $\mathcal{L}_{3,1}^{E}$ and $\mathcal{L}_{3,2}^{E}$ also have expressions, analogous to formula (28) for $D^{E}$, as linear combinations of the Kronecker-Eisenstein series $\sum_{\omega} \frac{\chi(\omega)}{\omega^{2} \bar{\omega}^{2}}$ and $\sum_{\omega} \frac{\chi(\omega)}{\omega \bar{\omega}^{3}}$, where $\omega$ runs over the lattice $L$ defining $E=\mathbb{C} / L$ and $\chi: L \rightarrow S^{1}$ is the character corresponding to $P$. There are of course also higher elliptic polylogarithms, relevant for the conjectural formula for $L\left(\operatorname{Sym}^{m-1}(E), m\right)$, which can be expressed either as averages over $q^{\mathbb{Z}}$ of higher classical polylogarithmic functions or else as linear combinations of higher Kronecker-Eisenstein series $\sum \chi(\omega) / \omega^{a} \bar{\omega}^{b}$. These functions, for which explicit formulas can be found in [54], are the elliptic analogues of the one-valued polylogarithm functions $\mathcal{L}_{m}: \mathbb{C} \rightarrow \mathbb{R}$. There are also elliptic analogues of the original many-valued polylogarithm function $L i_{m}$, studied in detail in the beautiful paper [32] of A. Levin.

We would like to characterize the conditions on $\xi=\sum n_{i}\left(P_{i}\right)$ that are needed in order for the image of $\xi$ under $\overrightarrow{\mathcal{L}}_{3}^{E}=\left(\mathcal{L}_{3}^{E}, J_{3}^{E}\right)$ to belong to the expected 2-dimensional lattice. Let us suppose for convenience that we are looking at a curve $E$ like $E_{37}$ for which the conditions (i)-(iii) of $\S 7$ are the correct ones for $D_{2}^{E}$. Then we find (both experimentally and based on the conjectural theory) that the conditions (i) and (ii) get replaced by

$$
\sum n_{i}=0 \in \mathbb{Z}, \quad \sum n_{i}\left(P_{i}\right)^{2}=0 \text { in } \operatorname{Sym}^{2}(E), \quad \sum n_{i}\left(P_{i}\right)^{4}=0 \text { in } \operatorname{Sym}^{4}(E),
$$

and the condition (iii) by

$$
\sum n_{i} \lambda_{p}\left(P_{i}\right)\left(P_{i}\right)^{2}=0 \text { in } \operatorname{Sym}^{2}(E) \text { for every prime } p .
$$


but there is a further condition, analogous to the one needed in the usual polylogarithm case. Namely, $\left(\mathrm{i}^{\prime}\right)$ and $\left(\mathrm{ii}^{\prime}\right)$ imply that $\iota_{\phi}(\xi)=\sum n_{i} \phi\left(P_{i}\right) P_{i}$ belongs to $\mathcal{A}_{2}(E / \mathbb{Q})$ for every homomorphism $\phi: E(\mathbb{Q}) \rightarrow \mathbb{Z}$; the further condition is then, that this element $\iota_{\phi}(\xi)$ maps to 0 in the Bloch group $\mathcal{B}_{2}=\mathcal{A}_{2} / \mathcal{C}_{2}$. All of the conditions together can be formulated more succinctly as saying that $\iota_{\phi}(\xi) \in \mathcal{C}_{2}(E / \mathbb{Q})$ for all homomorphisms $\phi: E(\overline{\mathbb{Q}}) \rightarrow \mathbb{Q}$.

Example. We again take $E_{37}$. We had found 4 elements $\xi_{3}, \xi_{4}, \xi_{6}$ and $\xi_{10}^{*} \in \mathcal{A}_{2}$ and by (30) three relations $4 \xi_{4}-13 \xi_{3}, 4 \xi_{6}-45 \xi_{3}, \xi_{10}^{*}-31 \xi_{3}$ in $\mathcal{C}_{2}$. "Pseudo-integrating" (i.e. replacing $\sum n_{i}(i P)$ by $\sum_{i \neq 0} \frac{n_{i}}{i}(i P)$, cf. Example 3 in $\left.\S 3\right)$ and then adding a "constant of integration" $n_{0}(O)$ to make the new divisor of degree 0 gives us three new elements

$$
\begin{aligned}
\eta_{4} & =3(4 P)-13(3 P)+18(2 P)-3(P)-5(O) \\
\eta_{6} & =2(6 P)-45(3 P)+60(2 P)+93(P)-110(O) \\
\eta_{10} & =3(10 P)-24(5 P)-310(3 P)+585(2 P)+750(P)-1004(O),
\end{aligned}
$$

which satisfy all the above conditions and are linearly independent. If the theory is correct, then the images of these three divisors under the map $\overrightarrow{\mathcal{L}}_{3}^{E}$ should lie in a 2-dimensional lattice with a covolume related to $L\left(\operatorname{Sym}^{2}(E), 3\right)$. Evaluating $\mathcal{L}_{3,1}^{E}$ and $\mathcal{L}_{3,2}^{E}$ numerically, we get the values

$$
\begin{aligned}
\overrightarrow{\mathcal{L}}_{3}^{E}\left(\eta_{4}\right) & =(-31.93647324246920545 \ldots, 51.36032576407323769 \ldots), \\
\overrightarrow{\mathcal{L}}_{3}^{E}\left(\eta_{6}\right) & =(-270.92487957661031873 \ldots, 29.85229569612024863 \ldots), \\
\overrightarrow{\mathcal{L}}_{3}^{E}\left(\eta_{10}\right) & =(-2374.45096970455445769 \ldots, 165.95000973693576237 \ldots),
\end{aligned}
$$

and indeed we find a linear combination $\eta_{10}-9 \eta_{6}+2 \eta_{4}$ which maps under $\overrightarrow{\mathcal{L}}_{3}^{E}$ to $(0,0)$ to many decimal places. The determinant formed of the values of $\overrightarrow{\mathcal{L}}_{3}^{E}$ for $\eta_{4}$ and $\eta_{6}$ is

$$
\operatorname{Reg}_{3}(E)=\left|\begin{array}{ll}
\mathcal{L}_{3,1}^{E}\left(\eta_{4}\right) & \mathcal{L}_{3,2}^{E}\left(\eta_{4}\right) \\
\mathcal{L}_{3,1}^{E}\left(\eta_{6}\right) & \mathcal{L}_{3,2}^{E}\left(\eta_{6}\right)
\end{array}\right|=12961.41302992157 \ldots
$$

On the other hand, we can compute

$$
L\left(\operatorname{Sym}^{2}(E), 3\right)=1.526262007533073 \ldots
$$

(see below) and we find, within the precision of the calculation, the relation

$$
\operatorname{Reg}_{3}(E)=\frac{37^{3}}{4} \Im(\tau)^{2} L\left(\operatorname{Sym}^{2}(E), 3\right) .
$$

Computing the $L$-value. We end by explaining how to compute $L\left(\operatorname{Sym}^{2}(E), 3\right)$. Let $f=\sum a_{n} q^{n} \in S_{2}\left(\Gamma_{0}(N)\right)$ be the modular form corresponding to $E$. Define the Dirichlet series $L_{2}(f, s)$ by

$$
L_{2}(f, s):=\zeta(s-1) L\left(\operatorname{Sym}^{2}(E), s\right)=\sum_{n=1}^{\infty} \frac{b_{n}}{n^{s}},
$$

where the coefficients $b_{n}$ can be obtained by

$$
L_{2}(f, s)=\left(1+N^{1-s}\right) \zeta(2 s-2) \sum_{n=1}^{\infty} \frac{a_{n}^{2}}{n^{s}} .
$$


(This formula is for the $N=37$ case; for other $f$ the Euler factors for the primes of bad reduction might change.) The function $L_{2}(f, s)$ can be shown by Rankin's method to have the functional equation $L_{2}^{*}(f, s)=L_{2}^{*}(f, 3-s)$, where $L_{2}^{*}(f, s)=(2 \pi)^{-2 s} N^{s} \Gamma(s) \Gamma(s-1) L_{2}(f, s)$. Hence, using the integral representation of $\Gamma(s) \Gamma(s-1)$ as the Mellin transform of the $K$-Bessel function $K_{1}(t)$ and splitting up the resulting integral into two pieces in the usual way, we find

$$
L_{2}(f, 3)=C\left(A-\frac{A^{2}}{2}\right)+\frac{1}{16} \sum_{n=1}^{\infty} \frac{b_{n}}{n^{3}} G_{1}\left(4 \pi \sqrt{\frac{n A}{N}}\right)+\frac{2^{8} \pi^{6}}{N^{3}} \sum_{n=1}^{\infty} b_{n} G_{2}\left(4 \pi \sqrt{\frac{n}{N A}}\right),
$$

for any $A>0$, where $C=2 \pi^{2} N^{-1} L\left(\operatorname{Sym}^{2}(E), 2\right)=1.3296021859908 \ldots$ and $G_{1}$ and $G_{2}$ are the functions

$$
G_{1}(x)=\int_{x}^{\infty} t^{4} K_{1}(t) d t, \quad G_{2}(x)=\int_{x}^{\infty} t^{-2} K_{1}(t) d t,
$$

both of which are bounded by a power of $x$ times $e^{-x}$ for $x$ large. The numerical value of $C$ can be obtained either from this formula (by evaluating it for two different values of $A$ or by taking its derivative, e.g. at $A=1)$ or, by Rankin's method, as a simple multiple $\left(=(2 \pi)^{3} / N^{2}\right)$ of the covolume of the lattice corresponding to $E$. The function $G_{1}(x)$ can be expressed in terms of Bessel functions by

$$
G_{1}(x)=\left(x^{4}+8 x^{2}\right) K_{0}(x)+\left(4 x^{3}+16 x\right) K_{1}(x)
$$

and hence can be computed numerically using standard software packages. For $G_{2}(x)$ we can use the rapidly convergent power series expansion

$$
\begin{aligned}
G_{2}(x)= & \frac{1}{2 x^{2}}-\frac{1}{4} \log ^{2}(x)-\frac{1}{2}\left(\gamma-\log 2-\frac{1}{2}\right) \log x+C_{0} \\
& -\frac{1}{4} \sum_{n=1}^{\infty} \frac{(x / 2)^{2 n}}{n(n+1) ! n !}\left(\log \frac{x}{2}+\gamma-\sum_{k=1}^{n} \frac{1}{k}-\frac{1}{2 n}-\frac{1}{2(n+1)}\right)
\end{aligned}
$$

(where $\gamma$ is Euler's constant and $C_{0}=-0.3629591663513 \ldots$ ) for reasonably small $x$ and the asymptotic expansion

$$
G_{2}(x) \sim \sqrt{\frac{\pi}{2 x}} e^{-x}\left(\frac{1}{x^{2}}-\frac{17}{8 x^{3}}+\frac{937}{128 x^{4}}-\cdots+\frac{r_{n}}{x^{n+2}}+\ldots\right)
$$

(where $r_{-1}=0, r_{n}+\left(n+\frac{3}{2}\right) r_{n-1}=\left(-\frac{1}{2}\right)^{n} n !\left(\begin{array}{c}1 / 2 \\ n\end{array}\right)\left(\begin{array}{c}-3 / 2 \\ n\end{array}\right)$ for $\left.n \geq 0\right)$, for large $x$. 


\section{REFERENCES}

1. Bailey, D.; Broadhurst, D., A seventeenth-order polylogarithm ladder, preprint (1999).

2. Beilinson, A., Higher regulators of modular curves, Applications of algebraic $K$-theory to algebraic geometry and number theory, Part I, II (Boulder, Colo., 1983), Contemp. Math. 55 (1986), Amer. Math. Soc., Providence, R.I., 1-34.

3. Beilinson, A.; Deligne, P., Interprétation motivique de la conjecture de Zagier reliant polylogarithmes et régulateurs, Proc. Sympos. Pure Math. 55, Part 2 (1994), Amer. Math. Soc., Providence, RI, 97-121.

4. Beilinson, A.; Deligne, P., Motivic polylogarithm and Zagier conjecture, preprint (1992).

5. Bloch, S., Applications of the dilogarithm function in algebraic K-theory and algebraic geometry, Proceedings of the International Symposium on Algebraic Geometry (Kyoto Univ., Kyoto, 1977) (1978), Kinokuniya Book Store, Tokyo, 103-114.

6. Bloch, S., Irvine lecture notes.

7. Bloch, S.; Grayson, D., $K_{2}$ and L-functions of elliptic curves: computer calculations, Applications of algebraic $K$-theory to algebraic geometry and number theory, Part I, II (Boulder, Colo., 1983), Contemp. Math. 55 (1986), Amer. Math. Soc., Providence, R.I., 79-88.

8. Böhm, J., Inhaltsmessung im $\mathbf{R}^{5}$ konstanter Krümmung, Arch. Math. 11 (1960), 298-309.

9. Borel, A., Cohomologie de $S L_{n}$ et valeurs de fonctions zêta aux points entiers, Ann. Scuola Norm. Sup. Pisa Cl. Sci. (4) 4 (1977), 613-636.

10. Boyd, D., Mahler's measure and special values of L-functions, Experiment. Math. 7 (1998), 37-82.

11. Browkin, J.; Gangl, H., Tame and wild kernels of quadratic imaginary number fields, Math. of Comp. 68 (1999), 291-305.

12. Chinburg, T., Salem numbers and L-functions, J. Number Theory 18 (1984), 213-214..

13. Cohen, H.; Lewin, L.; Zagier, D., A sixteenth-order polylogarithm ladder, Experiment. Math. 1 (1992), $25-34$.

14. Deninger, C., Deligne periods of mixed motives, K-theory and the entropy of certain $\mathbf{Z}^{n}$-actions, J. Amer. Math. Soc. 10 (1997), 259-281.

15. Deninger, C., Higher regulators and Hecke L-series of imaginary quadratic fields I, Invent. Math. 96 (1989), $1-69$.

16. Deninger, C., Higher regulators and Hecke L-series of imaginary quadratic fields II, Ann. of Math. (2) 132 (1990), 131-158.

17. de Jeu, R., Zagier's conjecture and wedge complexes in algebraic K-theory, Compositio Math. 96 (1995), 197-247.

18. Dupont, J.-L.; Sah, C. H., Scissors congruences. II, J. Pure Appl. Algebra 25 (1982), 159-195.

19. Erdős, P.; Stewart, C.; Tijdeman, R., Some diophantine equations with many solutions, Comp. Math. 66 (1988), 36-56.

20. Gangl, H., Funktionalgleichungen von Polylogarithmen, Bonner Math. Schriften 278 (1995).

21. Gangl, H., Computations in weight 4 motivic complexes, (to appear).

22. Gangl, H., A K-culator and $\zeta$-lyzer, (in preparation).

23. Goncharov, A.B., Geometry of configurations, polylogarithms, and motivic cohomology, Adv. Math. 114 (1995), 197-318.

24. Goncharov, A.B., Volumes of hyperbolic manifolds and mixed Tate motives, J. Amer. Math. Soc. 12 (1999), 569-618.

25. Goncharov, A.B., Mixed elliptic motives, Galois Representations in Arithmetic Algebraic Geometry. Edited by A.J. Scholl and R.L. Taylor, London Math. Soc. Lecture Note Series 243 (1998), Cambridge Univ. Press, 147-221.

26. Goncharov, A.B.; Levin, A.M., Zagier's conjecture on L(E,2), Invent. Math. 132 (1998), 393-432.

27. Gross, B., On the values of Artin L-functions, preprint, Brown University (1980).

28. Grunewald, F.; Kühnlein, S., On the proof of Humbert's volume formula, Manuscripta Math. 95 (1998), 431-436.

29. Kellerhals, R., Volumes in hyperbolic 5-space, Geom. Funct. Anal. 5 (1995), 640-667.

30. Kummer, E.E., Über die Transzendenten, welche aus wiederholten Integrationen rationaler Funktionen entstehen, J. Reine Angew. Math. 21 (1840), 74-90.

31. Lehmer, D.H., Factorization of certain cyclotomic functions, Ann. Math. 34 (1933), 461-479.

32. Levin, A.M., Elliptic polylogarithms: an analytic theory, Compositio Math. 106 (1997), 267-282.

33. Levin, A.M., Kronecker double series and the dilogarithm, preprint. 
34. Mahler, K., On some inequalities for polynomials in several variables, J. London Math. Soc. 37 (1962), 341-344.

35. Mestre, J.-F.; Schappacher, N., Séries de Kronecker et fonctions L des puissances symétriques de courbes elliptiques sur Q, Arithmetic algebraic geometry (Texel, 1989) (1991), Progr. Math. 89, Birkhäuser Boston, Boston, MA, 209-245.

36. Müller, P., Über Simplexinhalte in nichteuklidischen Räumen, Dissertation Bonn (1954).

37. Neumann, W. D.; Zagier, D., Volumes of hyperbolic three-manifolds, Topology 24 (1985), 307-332.

38. Ramakrishnan, D., Analogs of the Bloch-Wigner function for higher polylogarithms, Applications of algebraic $K$-theory to algebraic geometry and number theory, Part I, II (Boulder, Colo., 1983), Contemp. Math. 55 (1986), Amer. Math. Soc., Providence, R.I., 371-376.

39. Rohrlich, D.E., A modular version of Jensen's formula, Math. Proc. Cambridge Philos. Soc. 95 (1984), 15-20.

40. Rolshausen, K.; Schappacher, N., On the second K-group of an elliptic curve, J. Reine Angew. Math. 495 (1998), 61-77.

41. Schappacher, N.; Scholl, A., The boundary of the Eisenstein symbol, Math. Ann. 290 (1991), 303-321.

42. Smyth, C., On measures of polynomials in several variables, Bull. Austral. Math. Soc. 23 (1981), 49-63.

43. Stark, H., Values of L-functions at $s=1$. I-IV, Adv. in Math. 7 (1971), 301-343, 17 (1975), 60-92, 22 (1976), 64-84, 35 (1980), 197-235.

44. Suslin, A.A., $K_{3}$ of a field, and the Bloch group, Galois theory, rings, algebraic groups and their applications, Trudy Mat. Inst. Steklov 183 (1990), 180-199.

45. Tate, J., Les conjectures de Stark sur les fonctions $L$ d'Artin en $s=0$. (Lecture notes edited by D. Bernardi and N. Schappacher), Progress in Mathematics 47 (1984), Birkhäuser Boston, Inc., Boston, Mass.

46. Thurston, W., The geometry and topology of 3-manifolds, Chapter 7 "Computation of volume" by J. Milnor, Princeton Univ. Mimeographed Notes.

47. Thurston, W., Hyperbolic geometry and 3-manifolds, London Math. Soc. Lecture Note Ser., Low-dimensional topology (Bangor, 1979) 48 (1982), Cambridge Univ. Press, Cambridge-New York, 9-25.

48. Villegas, F. R., Modular Mahler measures I, Topics in number theory (University Park, PA, 1997) (1999), Math. Appl. 467, Kluwer Acad. Publ., Dordrecht, 17-48.

49. Wildeshaus, J., On an elliptic analogue of Zagier's conjecture, Duke Math. J. 87 (1997), 355-407.

50. Wojtkowiak, Z., A construction of analogs of the Bloch-Wigner function, Math. Scand. 65 (1989), 140-142.

51. Yoshida, T., The $\eta$-invariant of hyperbolic 3-manifolds, Invent. Math. 81 (1985), 473-514.

52. Zagier, D., Hyperbolic manifolds and special values of Dedekind zeta-functions, Invent. Math. 83 (1986), 285-301.

53. Zagier, D., Polylogarithms, Dedekind zeta functions and the algebraic $K$-theory of fields, Arithmetic algebraic geometry (Texel, 1989), Progr. Math. 89 (1991), Birkhäuser Boston, Boston, MA, 391-430.

54. Zagier, D., The Bloch-Wigner-Ramakrishnan polylogarithm function, Math. Ann. 286 (1990), $613-624$.

55. Zagier, D., Special values and functional equations of polylogarithms, Appendix A to "Structural properties of polylogarithms" (Leonard Lewin, ed.), Mathematical Surveys and Monographs 37 (1991), AMS, Providence, R.I., $377-400$. 